\title{
Reducing latency cost in 2D sparse matrix partitioning models ${ }^{\text {iर }}$
}

\author{
Oguz Selvitopi ${ }^{\mathrm{a}}$, Cevdet Aykanat ${ }^{\mathrm{a}, *}$ \\ Bilkent University, Computer Engineering Department, 06800, Ankara, Turkey
}

\section{A R T I C L E I N F O}

\section{Article history:}

Received 8 March 2015

Revised 11 December 2015

Accepted 21 April 2016

Available online 23 April 2016

\section{MSC:}

00-01

99-00,

\section{Keywords:}

Parallel iterative solvers

Nonsymmetric linear systems

Sparse matrix-vector multiplication

Sparse matrix partitioning

Latency overhead

Bandwidth overhead

\begin{abstract}
A B S T R A C T
Sparse matrix partitioning is a common technique used for improving performance of parallel linear iterative solvers. Compared to solvers used for symmetric linear systems, solvers for nonsymmetric systems offer more potential for addressing different multiple communication metrics due to the flexibility of adopting different partitions on the input and output vectors of sparse matrix-vector multiplication operations. In this regard, there exist works based on one-dimensional (1D) and two-dimensional (2D) fine-grain partitioning models that effectively address both bandwidth and latency costs in nonsymmetric solvers. In this work, we propose two new models based on 2D checkerboard and jagged partitioning. These models aim at minimizing total message count while maintaining a balance on communication volume loads of processors; hence, they address both bandwidth and latency costs. We evaluate all partitioning models on two nonsymmetric system solvers implemented using the widely adopted PETSc toolkit and conduct extensive experiments using these solvers on a modern system (a BlueGene/Q machine) successfully scaling them up to $8 \mathrm{~K}$ processors. Along with the proposed models, we put practical aspects of eight evaluated models (two 1D- and six 2D-based) under thorough analysis. To the best of our knowledge, this is the first work that analyzes practical performance of $2 \mathrm{D}$ models on this scale. Among evaluated models, the models that rely on 2D jagged partitioning obtain the most promising results by striking a balance between minimizing bandwidth and latency costs.
\end{abstract}

(C) 2016 Published by Elsevier B.V.

\section{Introduction}

Many scientific and engineering applications necessitate solving a linear system of equations. The methods used for this purpose are categorized as direct and iterative methods. When the linear system is large and sparse, iterative methods are preferred to their direct counterparts due to their speed and flexibility. Most widely used iterative methods for solving large-scale linear systems are based on Krylov subspace iterations.

A single iteration in Krylov subspace methods usually consists of one or more Sparse Matrix-Vector multiplications (SpMV), dot product(s) and vector updates. In a distributed setting, SpMV operations require regular or irregular point-to-point (P2P) communication depending on the sparsity pattern of the coefficient matrix in which each processor

\footnotetext{
This work was supported by The Scientific and Technological Research Council of Turkey (TÜBiTAK) under Grant EEEAG-114E545. This article is also based upon work from COST Action CA 15109 (COSTNET).

* Corresponding author. Tel.: +90 (312) 290 1213; fax: +90 (312) 2664047.

E-mail addresses: reha@cs.bilkent.edu.tr (O. Selvitopi), aykanat@cs.bilkent.edu.tr (C. Aykanat).
} 
sends/receives messages to/from a subset of processors. On the other hand, dot products necessitate global communication that involves a reduction operation on one or a few scalars in which all processors participate. Vector updates usually do not require any communication.

A common model to capture the cost of communicating a single message of $m$ words consists of two major components and is given by the formula $t_{s}+m t_{w}$. Here, $t_{s}$ is the startup time and signifies the costs related to preparation of the message (referred to as latency cost). The $t_{w}$ component is the time required to transfer a single word between two processors and is equal to the reciprocal bandwidth (referred to as bandwidth cost). Latency cost is proportional to the number of messages whereas bandwidth cost is proportional to the number of words. Among these two components, latency costs prove to be more vital for parallel performance as they are generally harder to avoid and improve [1]. Although both costs are reduced within time, the gap between them gradually increases in favor of bandwidth costs with an approximately $20 \%$ annual improvement over latency costs [1,2]. Furthermore, computation speeds evolve faster than communication speeds, making communication costs more critical for performance. With the latest developments in the scientific computing field, communication costs are likely to be a major factor in ranking fastest high performance computing (HPC) systems [3]. This work focuses on reducing latency costs of parallel SpMV operations in the context of nonsymmetric iterative solvers. The models and methods proposed in our work apply to matrices both with regular and irregular sparsity patterns. However, the benefits are more evident for the irregular ones, which are usually harder to exploit.

\subsection{Related work}

Communication requirements of iterative solvers have been of interest for more than three decades. There are numerous works on reducing communication overhead of global reduction operations in iterative solvers. Several works in this category aim at decreasing the number of global synchronization points in a single iteration of the solver by reformulating it [4-12]. Another important area of study is s-step Krylov subspace methods, which focus on further reducing the number of global synchronization points by a factor of $s$ by performing only a single reduction once in every $s$ iterations [8,13-17]. The performance gain of $s$-step methods comes at the cost of deteriorated stability and complications related to integration of preconditioners. However, these methods recently gained popularity again and promising studies address these shortcomings $[13,16,18,19]$. Another common technique is to overlap communication and computation with the aim of hiding global synchronization overheads [20,21]. Especially with the introduction of nonblocking collective constructs in the MPI-3 standard, this technique is gaining attraction [22-24]. Overlapping is commonly used for SpMV operations as well. In addition, a recent work proposed hierarchical and nested Krylov methods that constrain global reductions into smaller subsets of processors where they are cheaper [25]. Another recent work uses the idea of embedding SpMV communications into global reductions to avoid latency overhead of SpMV communications [26].

The performance of iterative solvers is also addressed by minimizing communication costs related to parallel SpMV operations, which is also addressed by this work. There are studies that can handle sparse matrices that are well-structured and have predictable sparsity patterns, generally arising from 2D/3D problems [16,27-29]. However, the studies in this field generally focus on combinatorial models that are capable of exploiting both regular and irregular patterns to obtain a good partition of the coefficient matrix. In this regard, graph and hypergraph partitioning models are widely utilized with successful partitioning tools such as MeTiS [30], PaToH [31], Scotch [32], Mondriaan [33]. These models can broadly be categorized as one-dimensional (1D) and two-dimensional (2D) partitioning models. In 1D models [30,31,34-39], each processor is responsible for a row/column stripe, whereas in 2D models, each processor may be responsible for a submatrix block (generally defined by a subset of rows and columns) or as in the most general case, each processor may be responsible for an arbitrarily defined subset of nonzeros. Compared to 1D models, 2D models possess more freedom in partitioning the coefficient matrix. Some works on 2D models do not take the communication volume into account, however they provide an upper bound on the number of messages communicated [40-44]. On the other hand, there are 2D models that aim at reducing volume, with or without providing a bound on the maximum number of messages [33,45-50]. 2D partitioning models in the literature can further be categorized into three classes: checkerboard partitioning [47,49,50] (also known as coarse-grain partitioning), jagged partitioning [45,49] and fine-grain partitioning [46,48,49]. Notably, a recent work [50] proposes a fast 2D partitioning for scale-free graphs via a two-phase approach. This method uses 1D partitioning to reduce volume in the first phase and an efficient heuristic in the second phase to obtain a bound on the maximum number of messages. This work differs from ours as it does not explicitly minimize the message count, instead, it uses a property of the Cartesian distribution of the matrices to provide the mentioned upper bound.

\subsection{Motivation and contributions}

Most of the aforementioned and other existing partitioning models optimize the objective of minimizing total communication volume, which is an effort to reduce bandwidth costs. However, communication cost is a function of both bandwidth and latency, with the latter being at least as important as the former, as the current trends indicate. The need for partitioning models that also consider other cost metrics has been noted in other works [26,34]. There are a few notable works that focus on different communication cost metrics. Balancing communication volume is one of them [33,51,52]. More important and overlooked work targets multiple communication metrics including latency [53], on which this study is based. Compared to [53], this study concentrates more on practical aspects. 
In this work, we claim and show that attempting to minimize a single communication objective hurts parallel performance and achieving a tradeoff between bandwidth and latency costs is the key factor for achieving scalability. The basic motivation is to employ a nonsymmetric partition in the solver. Note that in parallel SpMV operations of the form $w=A p$, one needs to partition the input vector $p$ and the output vector $w$ in addition to $A$. This can be achieved either by using a symmetric partition where the same partition is imposed on both input and output vectors, or by using a nonsymmetric partition where a distinct partition is employed for input and output vectors. The latter alternative is more appealing and it should be adopted whenever convenient since it is more flexible and allows operating in a broader search space. A nonsymmetric partition can be utilized in nonsymmetric linear system solvers such as the conjugate gradient normal equation error (CGNE) [54-56] and residual method (CGNR) [54-57], and the standard quasi-minimal residual (QMR) [58] where the coefficient matrix is square and nonsymmetric. The details of how to utilize nonsymmetric partitioning without incurring communication during linear vector update operations are explained for CGNE and CGNR solvers in Appendix E. We constrain ourselves to nonsymmetric square matrices in this work, but all proposed models apply to certain iterative methods that involve rectangular matrices as well.

Our work is based on [53], which also achieves a nonsymmetric partition through a two-phase methodology with a model called communication hypergraph. Our contributions and differences from [53] are as follows:

(i) We propose two new partitioning models for reducing latency which are based on 2D checkerboard and jagged partitioning. These models aim at reducing latency costs usually at the expense of increasing bandwidth costs. Similar models have been investigated [48,53], but they are based on 1D and 2D fine-grain models.

(ii) All proposed and investigated partitioning models are realized on two iterative methods CGNE and CGNR implemented with the widely adopted PETSc toolkit [59]. We describe how to obtain a nonsymmetric partition on the vectors utilized in these solvers using the communication hypergraph model and thoroughly evaluate partitioning requirements of them via experiments. In this manner, we differ from [53], in which the proposed methods were tested with a code developed by the authors that contains only parallel SpMV computations.

(iii) We conduct extensive experiments for the mentioned iterative solvers. Although better suited to large-scale systems, the communication hypergraph model was originally tested only for 24 processors on a local cluster and only for 1D partitioning. In this work, we test and show this model's validity on a modern HPC system (a BlueGene/Q machine) successfully scaling up to $8 \mathrm{~K}$ processors.

(iv) We compare one 1D-based, three 2D-based models (checkerboard, jagged and fine-grain), and these four models' latency-improved versions, making a total of eight partitioning models. Among these, the 2D models are somewhat overlooked in the literature, never being tested in a realistic setting on a large-scale system. Although their theoretical merits are of no question, their practical merits are not appreciated. In our experiments, we put these methods' practical aspects into a thorough analysis. The experiments show surprising results with $2 \mathrm{D}$ jagged partitioning and its latency-improved version performing better in the majority of the matrices.

The rest of the paper is organized as follows:Section 2.1 and 2.2 describe the proposed partitioning models to reduce the latency overhead of checkerboard and jagged models, respectively. These two sections describe basic checkerboard and jagged models as well. We also briefly review the fine-grain model and its latency-improved version in Section 2.3, since they are included in our experiments. We compare communication properties of all partitioning models in Section 3. Section 4 contains the results and discussions of the extensive large-scale experimental evaluation of eight partitioning models on a BlueGene/Q system with 28 matrices. Our experiments range from 256 to 8192 processors. Final remarks are given in Section 5. The presentation of the paper relies on the assumption that the reader is already familiar with the communication hypergraph model for 1D partitioning [53]. The unfamiliar reader can find a detailed background with explanatory examples about the communication hypergraph model in Appendix D.

\section{Reducing latency cost in $2 \mathrm{D}$ partitioning models}

2D models work at a finer level of partitioning granularity compared to 1D models by allowing nonzeros of a single row/column to be assigned to more than one processor. In this manner, they possess more flexibility in partitioning since they do not constrain the search space by assigning all nonzeros of a row/column to the same processor. This leads them to exploit existing partitioning tools better. 1D models necessitate a row-parallel/column-parallel algorithm (Appendix B), whereas 2D models necessitate a row-column-parallel algorithm. The fundamental difference between them is that the former necessitates a single communication stage in parallel SpMV operations, which is either pre-communication if the matrix is partitioned row wise, or post-communication if the matrix is partitioned column wise, whereas the latter necessitates two distinct communication stages: one before the local SpMV computations (on the input vector in a pre-communication stage via expand communication tasks) and one after the local SpMV computations (on the output vector in a post-communication stage via fold communication tasks), For more details on implementation issues regarding 2D partitioning, see [49].

In this section, we describe how to reduce latency overhead of 2D checkerboard and jagged partitioning models. For these models, we assume a $K=P \times Q$ virtual processor mesh. A simple example depicting a matrix partitioned with these two models is given in Fig. 1. Compared to their original counterparts, the proposed models are likely to increase the bandwidth costs by increasing communication volume. However, this issue is addressed by maintaining a balance on this metric. We also briefly review the 2D fine-grain model and compare it to checkerboard and jagged models as we evaluate it in our 


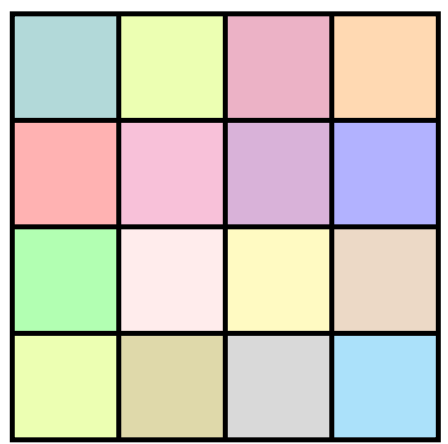

(a) Checkerboard

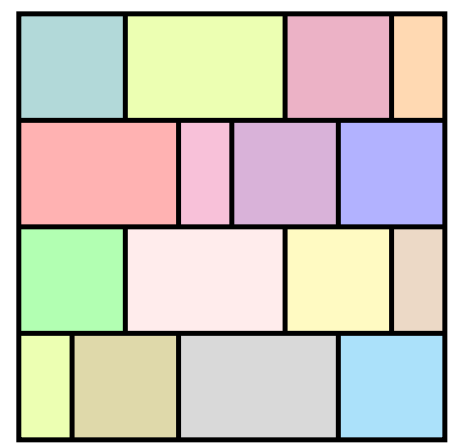

(b) Jagged

Fig. 1. A sample of $2 \mathrm{D}$ checkerboard and jagged partitionings on a $16=4 \times 4$ virtual processor mesh.

experiments. Note that a model to reduce latency overhead of fine-grain partitioning has already been investigated [48]. All proposed models are discussed on parallel $w=A p$. However, the arguments are also valid for $z=A^{T} r$ as communication requirements of $w=A p$ and $z=A^{T} r$ are the dual of each other and minimizing the objective function in $w=A p$ is equivalent to minimizing the objective function in $z=A^{T}$.

\subsection{Checkerboard partitioning}

Checkerboard partitioning is a two-phase process in which each phase utilizes a 1D partitioning model. The second phase depends on the first phase by using information obtained in the first phase to determine multiple vertex weights utilized in the second phase. For the rest of the section, we assume a 1D row wise partition in the first phase and a 1D column wise partition in the second phase. For the arguments made in this section, an analogous discussion holds for the dual scheme as well.

Consider a $K=P \times Q$ processor mesh and an $n \times n$ square matrix $A$. In the first phase, the column-net hypergraph model $\mathcal{H}_{\mathcal{R}}=\left(\mathcal{V}_{\mathcal{R}}, \mathcal{N}_{\mathcal{C}}\right)$ is used to obtain a $P$-way partition $\Pi_{\mathcal{R}}=\left\{\mathcal{V}_{1}, \ldots, \mathcal{V}_{P}\right\}$, which induces a $P$-way row wise partition $\left\{\mathcal{R}_{1}, \ldots, \mathcal{R}_{P}\right\}$ of $A$. Here, $\mathcal{R}_{\alpha}$ denotes the set of rows that correspond to vertices in $\mathcal{V}_{\alpha}$, for $\alpha=1, \ldots, P$. The rows in $\mathcal{R}_{\alpha}$ form a row stripe $A_{\alpha}$ whose size is $n_{\alpha} \times n$, with $n_{\alpha}=\left|\mathcal{V}_{\alpha}\right|$. At the end of the first phase, the assignment of rows of $A$ is determined by associating row stripe $A_{\alpha}$ with the $Q$ processors in row $\alpha$ of the processor mesh, $P_{\alpha, *}$.

In the second phase, the row-net hypergraph model $\mathcal{H}_{\mathcal{C}}=\left(\mathcal{V}_{\mathcal{C}}, \mathcal{N}_{\mathcal{R}}\right)$ is used to obtain a $Q$-way partition $\Pi_{\mathcal{C}}=\left\{\mathcal{V}_{1}, \ldots \mathcal{V}_{\mathcal{Q}}\right\}$, which induces a $Q$-way column wise partition $\left\{\mathcal{C}_{1}, \ldots, \mathcal{C}_{Q}\right\}$ of $A$. Here, $\mathcal{C}_{\beta}$ denotes the set of columns that correspond to vertices in $\mathcal{V}_{\beta}$, for $\beta=1, \ldots, Q$. The columns in $\mathcal{C}_{\beta}$ form column stripe $A_{\beta}$ whose size is $n \times n_{\beta}$, with $n_{\beta}=\left|\mathcal{V}_{\beta}\right|$. At the end of the second phase, we complete the assignment of columns of $A$ and actually obtain a $Q$-way column wise partition of each row stripe $A_{\alpha}$, forming $Q$ submatrix blocks $A_{\alpha, 1}, \ldots, A_{\alpha, Q}$. Hence, $\left(\Pi_{\mathcal{R}}, \Pi_{\mathcal{C}}\right)$ defines an assignment for rows and columns of $A$ where processor $P_{\alpha, \beta}$ is responsible for the set of rows in $\mathcal{R}_{\alpha}$ and the set of columns in $\mathcal{C}_{\beta}$. In other words, nonzero $a_{i j}$ is assigned to $P_{\alpha, \beta}$ if $r_{i} \in \mathcal{R}_{\alpha}$ and $c_{j} \in \mathcal{C}_{\beta}$.

This two-phase process aims at minimizing total communication volume for the pre- and post-communication stages in the first and second phases, respectively, while maintaining computational load balance [47,49]. A notable property of checkerboard partitioning is that it confines the communication in expand and fold operations to the processors in the same column and row of the processor mesh, respectively. It achieves a Cartesian distribution of the matrix, in which each processor owns an intersection of a subset of rows and a subset of columns. A row (column) is said to be coherent if the nonzeros of this row (column) generate partial results for (require) the same $w$-vector ( $x$-vector) element. Consider a row $r_{i}$ that is assigned to $\mathcal{R}_{\alpha}$ at the end of the first phase. The coherency of this row is preserved at this point as it is modeled by $v_{i}$ in $\mathcal{H}_{\mathcal{R}}$. In the second phase, the nonzeros of this row can be distributed among $Q$ processors in row $\alpha$ of the processor mesh, which is also the case for all other rows in $\mathcal{R}_{\alpha}$. Hence, row coherency is respected in a coarse level by assigning nonzeros of rows in $\mathcal{R}_{\alpha}$ to the processors in the same row of the processor mesh, $P_{\alpha, *}$. A coarse level here implies that the nonzeros belonging to a subset of rows are distributed among the same subset of processors (in this case among $P$ processors in a specific column of the processor mesh). This provides the upper bound $Q-1$ on the number of messages communicated in the post-communication stage as there are $Q$ processors in row $\alpha$ of the processor mesh. With a similar argument, column coherency is also respected in a coarse level by assigning nonzeros of columns in $\mathcal{C}_{\beta}$ to the processors in the same column of the processor mesh, $P_{*, \beta}$. This provides the upper bound $P-1$ on the number of messages communicated in the pre-communication stage as there are $P$ processors in column $\beta$ of the processor mesh. Hence, the maximum number of messages handled by a single processor is bounded by $P+Q-2$. In checkerboard partitioning, the second phase is performed with $P$-way multi-constraint $[60,61]$ partitioning to balance computational loads. 


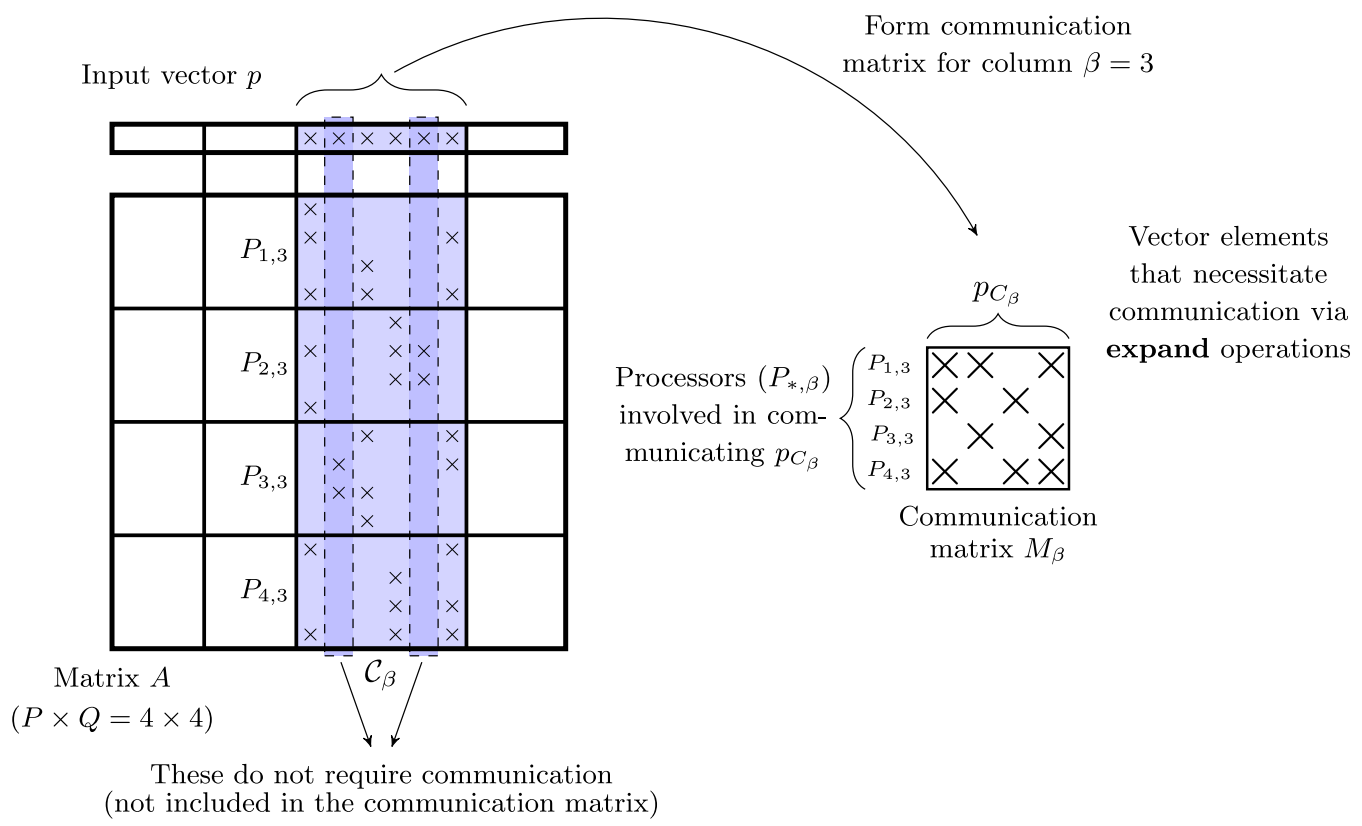

Fig. 2. Formation of the communication matrix for the third column of the processor mesh $(\beta=3)$ to summarize expand operations in the precommunication stage.

\subsubsection{Communication matrices}

The expand communication tasks in the checkerboard model are bound to distinct columns of the processor mesh. For this reason, to summarize the communication requirements of expand tasks in the pre-communication stage, we form $Q$ distinct communication matrices. Let $p_{C_{\beta}}$ denote the vector elements that necessitate communication in column $\beta$ of the processor mesh, for $1 \leq \beta \leq Q$. Note that at most $P$ processors can participate in communicating $p_{C_{\beta}}$, confined to the set of processors in $P_{*, \beta}$. We summarize the communication operations in column $\beta$ of the mesh with the $P \times\left|p_{C_{\beta}}\right|$ communication matrix $M_{\beta}$. Rows of $M_{\beta}$ correspond to processors in $P_{*, \beta}$ and columns of $M_{\beta}$ correspond to expand tasks on $p_{C_{\beta}}$. There exists a nonzero $m_{\alpha j} \in M_{\beta}$ if and only if there is a non-empty column segment in submatrix $A_{\alpha, \beta}$ at the respective column. The nonzeros in column $j$ of $M_{\beta}$ represent the set of processors that participate in communicating $p_{C_{\beta}}$ [j], which is a subset of processors in $P_{*, \beta}$. The nonzeros in row $\alpha$ of $M_{\beta}$ represent the expand tasks processor $P_{\alpha, \beta}$ participates in. Note that vector elements corresponding to internal columns (those which have a single non-empty column segment in $P$ row stripes) do not incur communication and they are not included in $M_{\beta}$. These vector elements should be assigned to the respective processors to avoid unnecessary communication. An example in Fig. 2 is presented to illustrate the formation of communication matrix $M_{\beta}$ for the third column of the processor mesh $(\beta=3)$ to summarize the expand tasks. There are four input vector elements that necessitate communication (denoted by $p_{C_{\beta}}$ ) and they form columns of $M_{\beta}$. For instance, the first column of $M_{\beta}$ has nonzeros corresponding to processors $P_{1,3}, P_{2,3}$ and $P_{4,3}$ since in matrix $A$, there exist nonzero column segments in the respective submatrix blocks. Two vector elements-second and fifth-corresponding to internal columns do not incur communication and they are not included in $M_{\beta}$; these elements should be assigned to $P_{3,3}$ and $P_{2,3}$, respectively, to avoid unnecessary communication.

The fold communication tasks in checkerboard model are bound to distinct rows of the processor mesh. Following a similar approach, we form $P$ distinct communication matrices to summarize the communication requirements of fold tasks in the post-communication stage. Let $w_{C_{\alpha}}$ denote the vector elements that necessitate communication in row $\alpha$ of the processor mesh, for $1 \leq \alpha \leq P$. We summarize the communication operations in row $\alpha$ of the mesh with the $\left|w_{C_{\alpha}}\right| \times Q$ communication matrix $M_{\alpha}$, where there exists a nonzero $m_{i \beta} \in M_{\alpha}$ if and only if there is a non-empty row segment in submatrix $A_{\alpha, \beta}$ at the respective row. An example is presented in Fig. 3 to illustrate the formation of communication matrix $M_{\alpha}$ in the third row of the processor mesh $(\alpha=3)$ to summarize the fold tasks. The dual of the discussions made for $M_{\beta}$ in Fig. 2 follows also for $M_{\alpha}$.

We form a total of $P+Q$ communication matrices to summarize communication requirements of checkerboard partitioning. We can address the communication requirements of both pre- and post-communication stages independently since communication operations in these stages are bound to distinct columns and rows of the processor mesh, respectively. Formation of these communication matrices is illustrated in Fig. 4. 


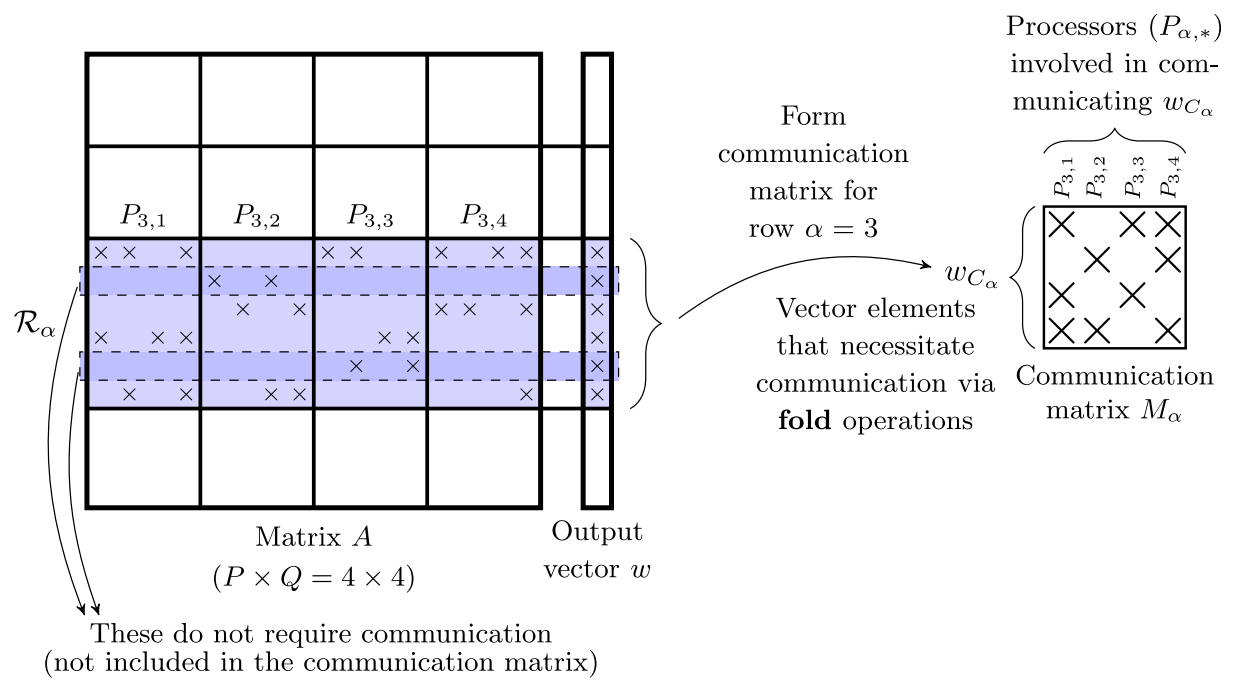

Fig. 3. Formation of the communication matrix for the third row of the processor mesh $(\alpha=3)$ to summarize fold operations in the post-communication stage.

\subsubsection{Formation of communication hypergraphs}

We form $Q$ hypergraphs from $Q$ communication matrices for the pre-communication stage. For each $M_{\beta}$, a communication hypergraph $\mathcal{H}_{\beta}^{C M}$ is formed using the row-net hypergraph model, for $1 \leq \beta \leq Q$. The net set of $\mathcal{H}_{\beta}^{C M}$ represents the processors in column $\beta\left(P_{*, \beta}\right)$ of the processor mesh and the vertex set of $\mathcal{H}_{\beta}^{C M}$ represents the expand tasks on $p_{C_{\beta}}$. Hence, there are $P$ nets and $\left|p_{C_{\beta}}\right|$ vertices in $\mathcal{H}_{\beta}^{C M}$. A vertex $v_{j}$ in $\mathcal{H}_{\beta}^{C M}$ is connected by the set of nets corresponding to processors that communicate the respective vector element $p_{c_{\beta}}[j]$. In all $Q$ communication hypergraphs, the total number of vertices is equal to $\left|p_{C}\right|=\sum_{\beta=1}^{Q}\left|p_{C_{\beta}}\right|$ and the total number of nets is equal to $Q \times P=K$.

In a similar manner, we form $P$ hypergraphs from $P$ communication matrices for the post-communication stage. For each $M_{\alpha}$, a communication hypergraph $\mathcal{H}_{\alpha}^{C M}$ is formed using the column-net hypergraph model, for $1 \leq \alpha \leq P$. The net set of $\mathcal{H}_{\alpha}^{C M}$ represents the processors in row $\alpha\left(P_{\alpha, *}\right)$ of the processor mesh and the vertex set of $\mathcal{H}_{\alpha}^{C M}$ represents the fold tasks on $w_{C_{\alpha}}$. Hence, there are $Q$ nets and $\left|w_{\mathcal{C}_{\alpha}}\right|$ vertices in $\mathcal{H}_{\alpha}^{C M}$. A vertex $v_{i}$ in $\mathcal{H}_{\alpha}^{C M}$ is connected by the set of nets corresponding to the processors that communicate the respective vector element $w_{C_{\alpha}}[i]$. In all $P$ communication hypergraphs, the total number of vertices is equal to $\left|w_{C}\right|=\sum_{\alpha=1}^{P}\left|w_{C_{\alpha}}\right|$ and the total number of nets is equal to $P \times Q=K$.

In total, we form $P+Q$ communication hypergraphs from $P+Q$ communication matrices. This process is illustrated in Fig. 4.

\subsubsection{Partitioning of the communication hypergraphs}

We partition $\mathcal{H}_{\beta}^{C M}$ to get a $P$-way partition $\Pi_{\beta}=\left\{\mathcal{V}_{1}, \mathcal{V}_{2}, \ldots, \mathcal{V}_{P}\right\}$ and obtain a distribution of expand tasks among $P$ processors in column $\beta$ of the processor mesh for the pre-communication stage, for $1 \leq \beta \leq Q$. The responsibility of the expand tasks represented by the vertices in $\mathcal{V}_{\alpha} \in \Pi_{\beta}$ is assigned to processor $P_{\alpha, \beta}$. Consider a net $n_{\alpha, \beta}$ in $\mathcal{H}_{\beta}^{C M}$ that represents $P_{\alpha, \beta}$. The connectivity set of this net contains the parts that correspond to the processors each of which send a message to $P_{\alpha, \beta}$. The size of this set can be at most $P$ since $\mathcal{H}_{\beta}^{C M}$ is partitioned into $P$, bounding the number of messages sent/received by a single processor by $P-1$ in the pre-communication stage. Hence, this feature of original checkerboard partitioning is still respected. In partitioning $\mathcal{H}_{\beta}^{\mathrm{CM}}$, the partitioning objective of minimizing cutsize corresponds to minimizing the number of messages communicated in column $\beta$ of the processor mesh in the pre-communication stage, and the partitioning constraint of maintaining balance among part weights corresponds to obtaining a balance on the communication volume loads of these processors.

Similarly, we partition $\mathcal{H}_{\alpha}^{C M}$ to get a $Q$-way partition $\Pi_{\alpha}=\left\{\mathcal{V}_{1}, \mathcal{V}_{2}, \ldots, \mathcal{V}_{Q}\right\}$ and obtain a distribution of fold tasks among $Q$ processors in row $\alpha$ of the processor mesh for the post-communication stage, for $1 \leq \alpha \leq P$. $\mathcal{H}_{\alpha}^{C M}$ is partitioned into $Q$ bounding the number of messages sent/received by a single processor by $Q-1$ in the post-communication stage. Hence, this feature of original checkerboard partitioning is also respected. The partitioning objective and the balancing constraint are identical to those in partitioning $\mathcal{H}_{\beta}^{C M}$.

The formed $P+Q$ hypergraphs can be independently partitioned since they do not depend on each other in any way. The maximum number of messages handled by a single processor is still $P+Q-2$ as in the original checkerboard partitioning. As a result, we improve latency costs in each row/column of the processor mesh independently while respecting basic characteristics of checkerboard partitioning. 


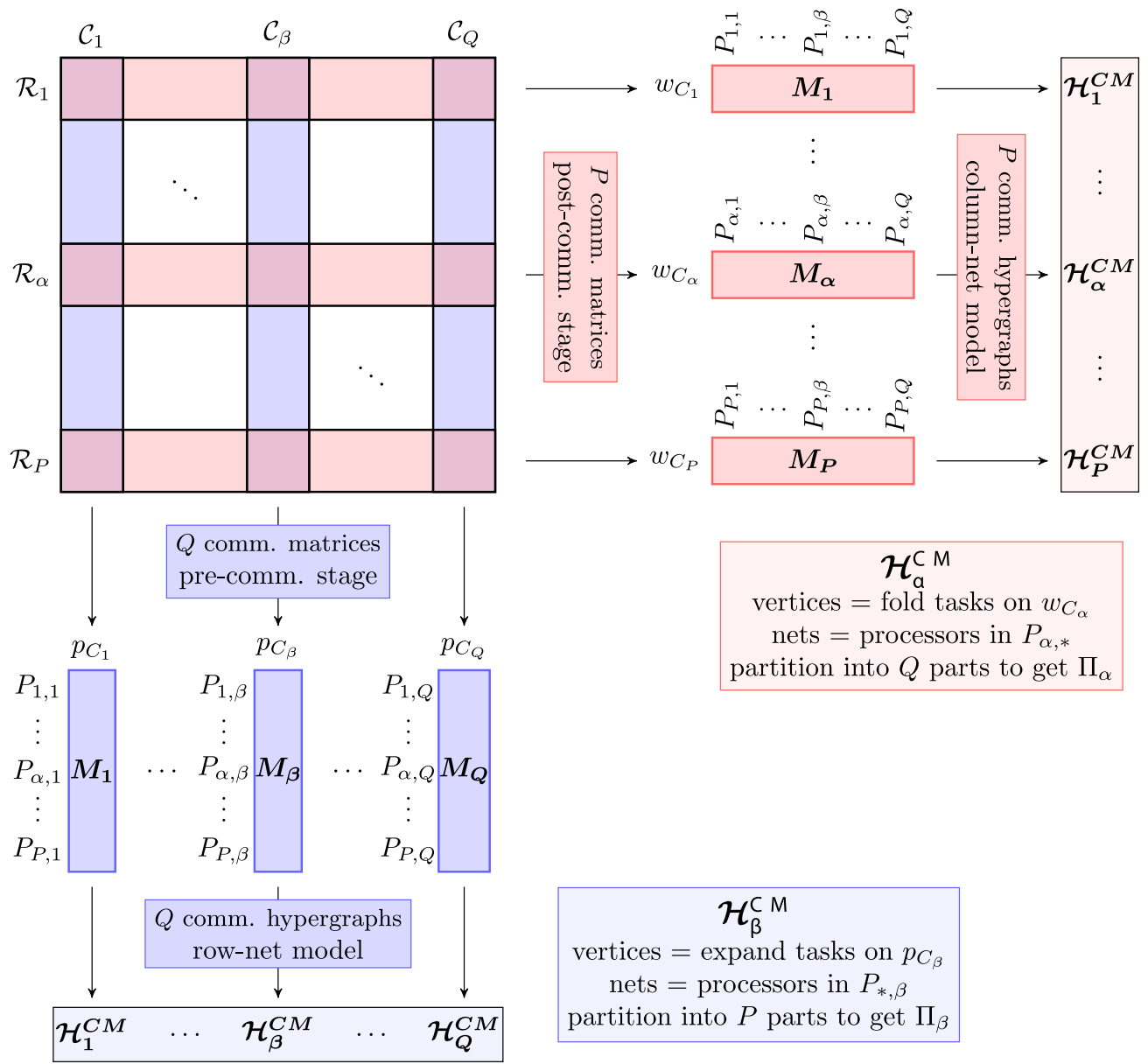

Fig. 4. Minimizing latency cost in checkerboard partitioning model.

\subsection{Jagged partitioning}

Jagged partitioning consists of two phases. The first phase consists of a single 1D partitioning model, whereas the second phase consists of multiple, independent and same type of 1D partitioning models. The second phase depends on the first phase by using the partitioning information obtained in the first phase to determine vertex sets and vertex weights for the models formed in the second phase. For the rest of the section, we assume a 1D row wise partition in the first phase and a 1D column wise partition in the second phase. For the arguments made in this section, an analogous discussion holds for the dual scheme as well.

Assume a $K=P \times Q$ processor mesh and an $n \times n$ square matrix $A$. The first phase of jagged partitioning is exactly the same as the first phase of checkerboard partitioning: a column-net hypergraph model $\mathcal{H}_{\mathcal{R}}=\left(\mathcal{V}_{\mathcal{R}}, \mathcal{N}_{\mathcal{C}}\right)$ is used to obtain a $P$-way partition $\Pi_{\mathcal{R}}=\left\{\mathcal{V}_{1}, \ldots, \mathcal{V}_{P}\right\}$, which induces a row wise partition $\left\{\mathcal{R}_{1}, \ldots, \mathcal{R}_{P}\right\}$ of $A$. At the end of this phase, the rows in row stripe $A_{\alpha}$ are associated with the $Q$ processors in row $\alpha$ of the processor mesh, $P_{\alpha, *}$.

In the second phase, we form a hypergraph $\mathcal{H}_{\alpha}$ for each row submatrix $A_{\alpha}$ obtained in the former phase using the rownet hypergraph model, for $1 \leq \alpha \leq P$. In total, $P$ hypergraphs are formed. In this aspect, jagged partitioning differs from checkerboard partitioning - which forms a single hypergraph in the second phase. The net set of $\mathcal{H}_{\alpha}$ represents rows of $A_{\alpha}$ and the vertex set of $\mathcal{H}_{\alpha}$ represents columns of $A$ that have a nonzero column segment in $A_{\alpha}$. Hence, the same vertex can appear in multiple hypergraphs since the corresponding column may have nonzero column segments in more than one row stripes. These $P$ hypergraphs are independently partitioned into $Q$ parts to obtain a $Q$-way partition of each row stripe. At the end of the second phase, for each row stripe $A_{\alpha}$, we obtain $Q$ submatrix blocks $A_{\alpha, 1}, \ldots, A_{\alpha, Q}$ by partitioning vertices corresponding to columns of $A_{\alpha}$.

The first and the second phase aim to minimize the volume of communication in pre- and post-communication stages, respectively, while maintaining computational load balance [47,49]. In contrast to checkerboard partitioning, the objective in the second phase of the jagged partitioning is addressed independently by partitioning row stripes separately in distinct hypergraphs. The jagged model also differs from the checkerboard model as it does not lead to a Cartesian distribution 
of the matrix. Jagged partitioning is more flexible in this sense since it allows nonzeros of a column to be distributed among any processor that is in distinct rows of the processor mesh - not just among the processors that are in the same column of the mesh, as is the case for checkerboard partitioning. Hence, the column coherency is not preserved in the assignment of columns. This leads to improved communication volume for expand tasks in the pre-communication stage at the expense of increasing the upper bound on the number of messages handled by a single processor. In other words, the jagged model sacrifices the coarse level coherency of columns and causes the number of messages handled by a single processor in the pre-communication stage to be at most $P \times Q-Q=K-Q$. In this stage, a processor may communicate with any other processor except the processors that are in the same row of the processor mesh as this processor. On the other hand, the coherency of rows owned by a processor is respected in a coarse level as in checkerboard partitioning. This is because nonzeros of these rows are distributed among processors in the same row of processor mesh, $P_{\alpha, *}$, if the respective processor is in row $\alpha$. Hence, the number of messages handled by a single processor for fold tasks in the post-communication stage is bounded by $Q-1$ as there are $Q$ processors in a single row of the processor mesh. Consequently, the maximum number of messages handled by a processor can be at most $(K-Q)+(Q-1)=K-1$ in jagged partitioning.

\subsubsection{Communication matrices}

The expand communication tasks in the jagged model are not bound to distinct columns of the processor mesh. For this reason, we form a single communication matrix to summarize the communication requirements of expand tasks in the precommunication stage. Let $p_{C}$ denote the vector elements that necessitate communication. We summarize the communication operations with the $(K=P \times Q) \times\left|p_{C}\right|$ communication matrix $M_{R}$. Rows of $M_{R}$ correspond to all processors and columns of $M_{R}$ correspond to expand tasks on $p_{C}$. Consider two vector elements owned by the same processor. Although these two elements can be communicated by at most $P$ processors (each of which belongs to a distinct row of the processor mesh), they do not necessarily need to be confined to the same column of the processor mesh. For this reason, we include all processors in $M_{R}$. The formation of $M_{R}$ essentially resembles that of $1 \mathrm{D}$ row-parallel $w=A p(\mathrm{D} .1): m_{k j} \neq 0$ if and only if column $c_{j}$ has a nonzero column segment in $k$ th row stripe of $A$. Nonzeros in column $c_{j} \in M_{R}$ represent the set of processors that participate in communicating $p_{C}[j]$ and nonzeros in row $r_{k} \in M_{R}$ represent the expand tasks $P_{k}$ participates in. The difference is, however, as a consequence of jagged partitioning, each column in $M_{R}$ can have at most $P$ nonzeros instead of $K$. As usual, vector elements corresponding to internal columns are not included in $M_{R}$ since they do not necessitate communication.

In contrast to expand tasks, the fold communication tasks are bound to distinct rows of the processor mesh. The communication requirements of fold tasks in the post-communication stage are thus summarized by $P$ distinct communication matrices, $M_{\alpha}$, for $1 \leq \alpha \leq P$. The formation of these matrices is the same as the formation of matrices for summarizing communication requirements of fold tasks in checkerboard partitioning. The semantics of nonzeros in rows and columns of $M_{\alpha}$ are identical to those of $M_{\alpha}$ in checkerboard partitioning.

We form a total of $P+1$ communication matrices to summarize communication requirements of jagged partitioning. We can address the communication requirements of the post-communication stage independently using $P$ matrices. However, since communication operations in the pre-communication stage are not bound to the processors in the same column of the processor mesh, the expand tasks are represented in a single matrix with all $K$ processors. Formation of these communication matrices is illustrated in Fig. 5.

\subsubsection{Formation of the communication hypergraphs}

For the pre-communication stage, we form a single communication hypergraph $\mathcal{H}_{R}^{C M}$ from communication matrix $M_{R}$ using the row-net hypergraph model. The net set of $\mathcal{H}_{R}^{C M}$ corresponds to $K$ processors and the vertex set of $\mathcal{H}_{R}^{C M}$ corresponds to expand tasks on $p_{C}$. Hence, there are $K$ nets and $\left|p_{C}\right|$ vertices in $\mathcal{H}_{R}^{C M}$. A vertex $v_{j}$ in $\mathcal{H}_{R}^{C M}$ is connected by the set of nets corresponding to processors that communicate the respective vector element $p_{C}[j]$. Note that $v_{j}$ can be connected by at most $P$ nets, i.e., $d_{j} \leq P$.

For the post-communication stage, we form $P$ communication hypergraphs from $P$ communication matrices. The formation of these communication hypergraphs is actually the same as for checkerboard partitioning. A communication hypergraph $\mathcal{H}_{\alpha}^{C M}$ is formed using the column-net hypergraph model for matrix $M_{\alpha}$, for $1 \leq \alpha \leq P$. The semantics of these hypergraphs are also the same: the net set of $\mathcal{H}_{\alpha}^{C M}$ represents the processors in row $\alpha$ of the processor mesh, $P_{\alpha, *}$, and the vertex set of $\mathcal{H}_{\alpha}^{C M}$ represents the fold tasks on $w_{C_{\alpha}}$. Similarly, there are $\left|w_{C}\right|$ vertices and $K$ nets in all communication hypergraphs.

In total, we form $P+1$ communication hypergraphs from $P+1$ communication matrices. This process is illustrated in Fig. 5.

\subsubsection{Partitioning of the communication hypergraphs}

Communication hypergraph $\mathcal{H}_{R}^{C M}$ is partitioned to obtain a $K$-way partition $\Pi_{R}=\left\{\mathcal{V}_{1}, \mathcal{V}_{2}, \ldots, \mathcal{V}_{K}\right\}$ to induce a distribution of expand tasks in the pre-communication stage. The responsibility of the expand tasks represented by the vertices in $\mathcal{V}_{k}$ is assigned to processor $P_{k}$. Consider a net $n_{k}$ in $\mathcal{H}_{R}^{C M}$ that represents $P_{k}$. The connectivity set of this net corresponds to processors each of which send a message to $P_{k}$. Since $\mathcal{H}_{R}^{C M}$ is partitioned into $K$ parts, the size of this set can be at most 


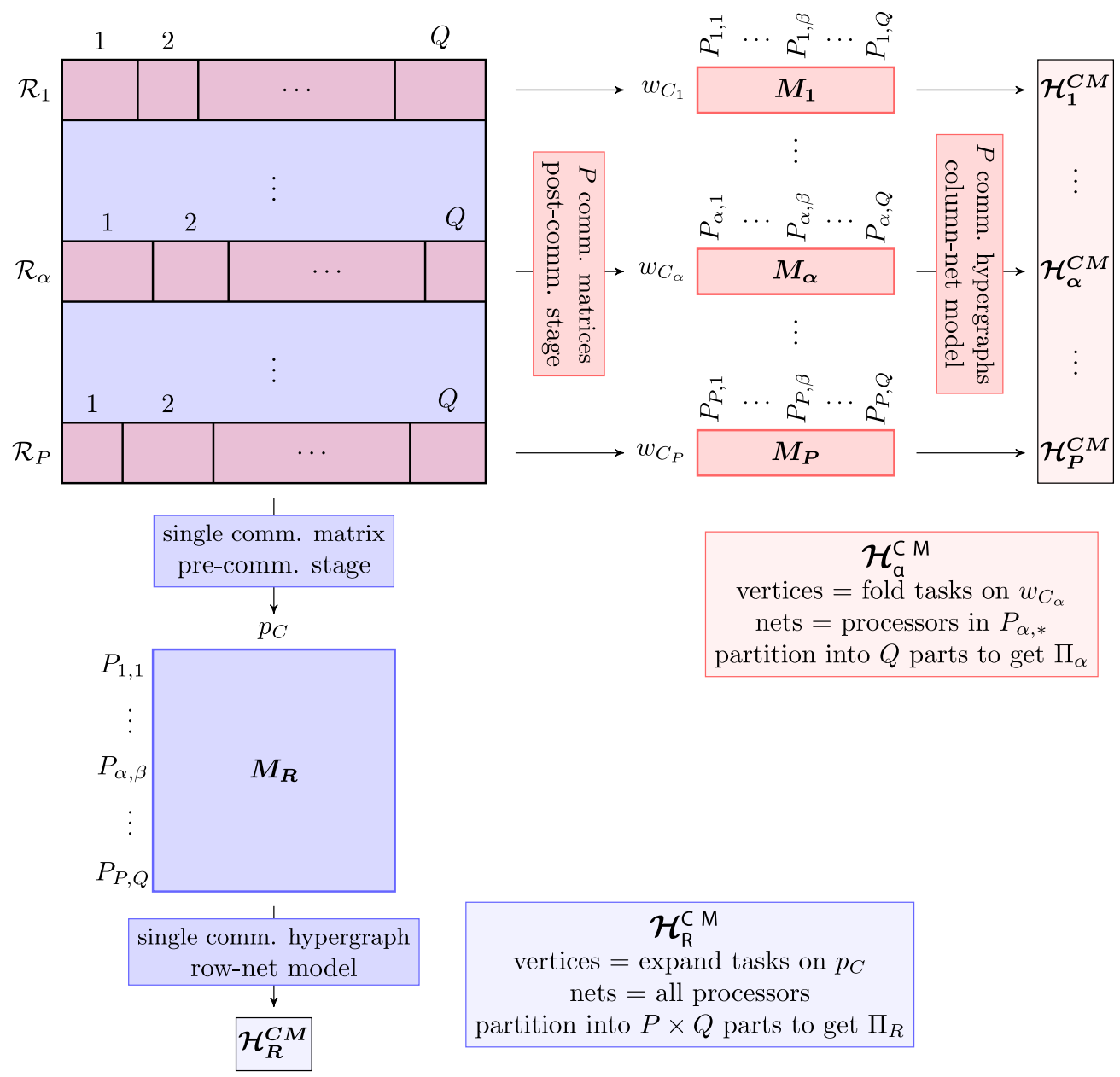

Fig. 5. Minimizing latency cost in jagged partitioning model.

$K$. Thus, the maximum number of messages sent/received by a single processor is $K-1$ in the pre-communication stage (note that it is $K-Q$ in the original jagged partitioning). In partitioning $\mathcal{H}_{R}^{C M}$, the partitioning objective of minimizing cutsize corresponds to minimizing the number of messages communicated in the pre-communication stage, and the partitioning constraint of maintaining balance among part weights corresponds to obtaining a balance on the communication volume loads of $K$ processors.

Communication hypergraph $\mathcal{H}_{\alpha}^{C M}$ is partitioned to obtain a $Q$-way partition $\Pi_{\alpha}=\left\{\mathcal{V}_{1}, \mathcal{V}_{2}, \ldots, \mathcal{V}_{Q}\right\}$, for $1 \leq \alpha \leq P$, to induce a distribution of fold tasks in the post-communication stage among $Q$ processors in row $\alpha$ of the processor mesh. The partitioning of these communication hypergraphs have the same semantics with those in checkerboard partitioning.

The formed $P+1$ hypergraphs can be partitioned independently, since they do not depend on each other in any way. Note that the maximum number of messages handled by a single processor is slightly increased from $K-1$ to $K+Q-2$, which is caused by the partitioning for distributing tasks in the pre-communication stage. However, the cases beyond this are expected to be rare as a good partitioning tool will avoid them. As a result, we improve latency costs while respecting most characteristics of the original jagged partitioning.

\subsection{Fine-grain (nonzero-based) partitioning}

We briefly review fundamental properties of fine-grain partitioning. This model is first proposed in [46] and its communication costs are improved in a later work using the CHG model [48]. Both of these models are included in our experiments.

The fine-grain model forms a hypergraph in which vertices represent nonzeros of $A$ and nets represent rows and columns of $A$. Nets corresponding to columns of $A$ capture the communication volume incurred in the pre-communication stage, while nets corresponding to rows of $A$ capture the communication volume incurred in the post-communication stage. Partitioning this hypergraph into $K$ parts induces a distribution of nonzeros of the matrix among $K$ processors. This leads to a completely arbitrary distribution of fine-grain computational tasks on a nonzero basis, where each vertex signifies a scalar 


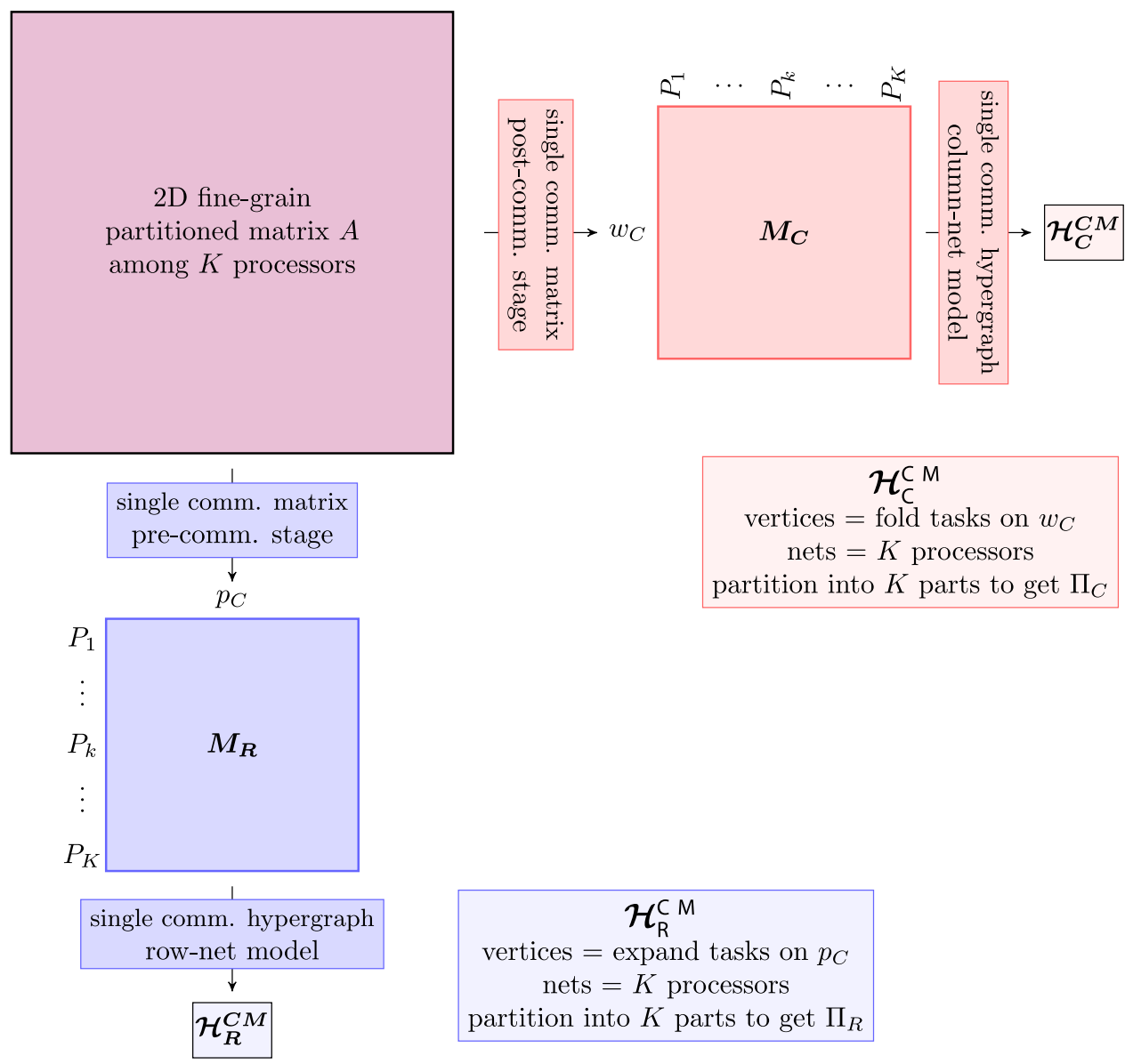

Fig. 6. Minimizing latency cost in fine-grain partitioning model.

multiplication with a single nonzero. Therefore, the fine-grain model respects neither row nor column coherence. In this aspect, it accommodates the highest level of flexibility by not restraining the computational tasks to coarser levels (i.e., nonzeros of a row and/or column) compared to checkerboard, jagged and 1D models. As a result, a processor may communicate with any other processor. Thus, the maximum number of messages handled by a single processor is $K-1$ in the pre-communication stage and is also $K-1$ in the post-communication stage, summing up to a total of $2(K-1)$ messages. The fine-grain model correctly minimizes the total communication volume while maintaining computational load balance. For more details, see [46,49].

The approach to improve communication requirements of the fine-grain model [48] consists of forming two communication matrices: one matrix for summarizing communication operations in the pre-communication stage and one matrix for summarizing communication operations in the post-communication stage. Compare this to the formation of communication matrices in the checkerboard and jagged models. We address the communication requirements in the checkerboard model by separately forming a total of $P+Q$ communication matrices since they are confined to distinct columns and rows of the processor mesh. Also in the jagged model, we form $P$ communication matrices for the post-communication stage. These are not valid for the fine-grain model since the resulting partition is arbitrarily defined on the nonzeros of the matrix and any of the $K$ processors may communicate with any other processor in both pre- and post-communication stages. For this reason, the whole set of processors and all vector elements that necessitate communication are included in two communication matrices. The process for reducing the communication costs of the fine-grain model is illustrated in Fig. 6.

\section{Comparison of partitioning models}

We compare the basic properties of the investigated partitioning models to aid the discussions of the results in experiments. It is assumed that $P=Q=\sqrt{K}$ in the $P \times Q$ processor mesh for ease of presentation.

Fig. 7 compares the partitioning models in terms of flexibility they provide during partitioning. 1D models lie at the left extreme of the spectrum since they represent each row/column of the coefficient matrix with a single distinct vertex as an atomic task. This leads to the assignment of all nonzeros of a row/column to an individual processor as a whole. Hence, 1D 


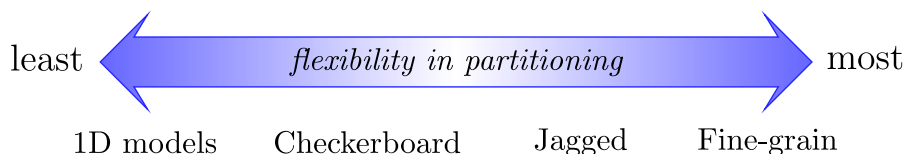

Fig. 7. Comparison of models in terms of partitioning flexibility.

Table 1

Comparison of partitioning models in terms of latency overhead and partitioning granularity.

\begin{tabular}{|c|c|c|c|c|c|}
\hline \multirow[t]{2}{*}{ Number of messages } & \multirow[t]{2}{*}{ Comm. stage } & \multirow[t]{2}{*}{$1 \mathrm{D}$} & \multicolumn{3}{|l|}{ 2D partitioning models } \\
\hline & & & Checkerboard & Jagged & Fine-grain \\
\hline \multirow{3}{*}{$\max$} & pre & $K-1$ & $\sqrt{K}-1$ & $K-\sqrt{K}$ & $K-1$ \\
\hline & post & $\mathrm{N} / \mathrm{A}$ & $\sqrt{K}-1$ & $\sqrt{K}-1$ & $K-1$ \\
\hline & overall & $K-1$ & $2(\sqrt{K}-1)$ & $K-1$ & $2(K-1)$ \\
\hline total & overall & $K(K-1)$ & $2 K(\sqrt{K}-1)$ & $K(K-1)$ & $2 K(K-1)$ \\
\hline Row/column coherency & & Either entire row or & Coarse-level on both & Coarse-level on either & None \\
\hline
\end{tabular}

models respect row/column coherency at the individual processor level. The fine-grain model lies at the right extreme of the spectrum since in this model each vertex represents an atomic task corresponding to a single nonzero of the matrix. This is the most flexible and the finest level of partitioning granularity available, where neither row nor column coherency is preserved. So, in theory, nonzeros of a row/column can be distributed among $K$ processors. Between these two extremes, the checkerboard and jagged models strive to distribute nonzeros of a row/column among a subset of processors. By doing so, they obtain a coarse-level row/column coherency at the processor mesh's row/column level. The checkerboard model leverages a coarse-level coherency in both partitioning phases whereas the jagged model leverages it in a single partitioning phase.

Among these partitioning models, 2D models are expected to achieve lower bandwidth costs compared to 1D models since they offer more flexibility in optimizing the objective of minimizing total communication volume. Among 2D models, fine-grain is expected to obtain the best results in terms of bandwidth costs, whereas checkerboard is likely to obtain the worst. The metrics related to latency costs (as upper bounds on the maximum number of messages) are presented in Table 1. Checkerboard has the lowest overhead with $2(\sqrt{K}-1)$ maximum messages per processor, whereas fine-grain has the highest overhead with $2(K-1)$. Although 1D and jagged models have the same upper bound $K-1$, in practice jagged partitioning is more likely to achieve better results in this metric since it restricts the number of messages in both stages of communication.

The discussions made so far in this section reflect the characteristics of the original partitioning models in which the communication hypergraph model is not used. The original models completely focus on minimizing bandwidth costs, disregarding latency-related objectives. Using the communication hypergraph model as a further step reduces latency costs at the expense of increasing bandwidth costs while respecting certain characteristics of the original models as much as possible. Our experimental evaluation shows that latency should definitely be on the table to achieve scalable performance.

\section{Experiments}

We evaluate two 1D-based and six 2D-based models, that is, a total of eight partitioning models. The evaluated models are based on 1D row wise partitioning (1D), checkerboard partitioning (CKBD), jagged partitioning (JGD) and fine-grain partitioning (FG). Four of the evaluated models are the baseline models in which the communication tasks are assigned to processors using a simple heuristic that aims at balancing the communication volume loads while respecting total volume attained in the initial partitioning. This heuristic is also utilized in [53] and is an adaptation of the best-fit-decreasing heuristic used in solving the NP-hard $K$-feasible Bin Packing (BP) problem [62]. These BP-enhanced baseline models are referred to as $1 D+B P, C K B D+B P, J G D+B P$ and $F G+B P$. These four baseline models aim to reduce two important volumerelated communication cost metrics, namely total volume and maximum volume. The remaining four evaluated models are the CHG-enhanced versions in which the communication tasks are assigned to processors using the communication hypergraph model. These CHG-enhanced models are referred to as $1 \mathrm{D}+\mathrm{CHG}, \mathrm{CKBD}+\mathrm{CHG}, \mathrm{JGD}+\mathrm{CHG}$ and FG+CHG. These aim to reduce total message count and maximum volume. Hence, we evaluate the merit of reducing a latency-related cost metric in partitioning. We use $\mathrm{PaToH}[31,61]$ to partition the computational hypergraphs formed in the first phase of all models and the communication hypergraphs formed in the second phase of the CHG-enhanced models.

We implemented CGNE and CGNR solvers via the PETSc toolkit [59] and utilized the mentioned models for partitioning the coefficient matrix and vectors in these solvers. Since obtained runtime results for both solvers are similar, we only present speedup results corresponding to CGNR. Note that the metrics regarding partitioning models (Section 4.1) such as total volume, message count, etc. are the same for both solvers as they contain the same type of communication operations. 
Table 2

Test matrices and their properties.

\begin{tabular}{|c|c|c|c|c|c|c|c|}
\hline \multirow[b]{3}{*}{ Matrix } & \multirow{2}{*}{\multicolumn{2}{|c|}{ Number of }} & \multicolumn{5}{|c|}{ Nonzeros } \\
\hline & & & \multirow[b]{2}{*}{ avg } & \multicolumn{2}{|c|}{ per row } & \multicolumn{2}{|c|}{ per column } \\
\hline & rows/cols & nonzeros & & $\min$ & $\max$ & $\min$ & $\max$ \\
\hline venkat01 & 62,424 & $1,717,792$ & 27.52 & 16 & 44 & 16 & 44 \\
\hline mc2depi & 525,825 & $2,100,225$ & 3.99 & 2 & 4 & 2 & 4 \\
\hline poisson3Db & 85,623 & $2,374,949$ & 27.74 & 6 & 145 & 6 & 145 \\
\hline thermomech_dK & 204,316 & $2,846,228$ & 13.93 & 7 & 20 & 7 & 20 \\
\hline stomach & 213,360 & $3,021,648$ & 14.16 & 7 & 19 & 6 & 22 \\
\hline FEM_3D_thermal2 & 147,900 & $3,489,300$ & 23.59 & 12 & 27 & 12 & 27 \\
\hline laminar_duct3D & 67,173 & $3,833,077$ & 57.06 & 1 & 89 & 3 & 89 \\
\hline xenon2 & 157,464 & $3,866,688$ & 24.56 & 1 & 27 & 1 & 27 \\
\hline iChem_Jacobian & 274,087 & $4,137,369$ & 15.10 & 5 & 17 & 5 & 17 \\
\hline torso3 & 259,156 & $4,429,042$ & 17.09 & 7 & 22 & 6 & 21 \\
\hline tmt_unsym & 917,825 & $4,584,801$ & 5.00 & 3 & 5 & 3 & 5 \\
\hline t2em & 921,632 & $4,590,832$ & 4.98 & 1 & 5 & 1 & 5 \\
\hline Hamrle3 & $1,447,360$ & $5,514,242$ & 3.81 & 2 & 6 & 2 & 9 \\
\hline largebasis & 440,020 & $5,560,100$ & 12.64 & 4 & 14 & 4 & 14 \\
\hline Chevron4 & 711,450 & $6,376,412$ & 8.96 & 2 & 9 & 2 & 9 \\
\hline cage13 & 445,315 & $7,479,343$ & 16.80 & 3 & 39 & 3 & 39 \\
\hline PR02R & 161,070 & $8,185,136$ & 50.82 & 1 & 92 & 5 & 88 \\
\hline atmosmodl & $1,489,752$ & $10,319,760$ & 6.93 & 4 & 7 & 4 & 7 \\
\hline kim2 & 456,976 & $11,330,020$ & 24.79 & 4 & 25 & 5 & 25 \\
\hline memchip & $2,707,524$ & $14,810,202$ & 5.47 & 2 & 27 & 1 & 27 \\
\hline Freescale1 & $3,428,755$ & $18,920,347$ & 5.52 & 1 & 27 & 1 & 25 \\
\hline circuit5M_dc & $3,523,317$ & $19,194,193$ & 5.45 & 1 & 27 & 1 & 25 \\
\hline fem_hifreq_circuit & 491,100 & $20,239,237$ & 41.21 & 12 & 110 & 12 & 110 \\
\hline rajat31 & $4,690,002$ & $20,316,253$ & 4.33 & 1 & 1252 & 1 & 1252 \\
\hline CoupCons3D & 416,800 & $22,322,336$ & 53.56 & 20 & 76 & 20 & 76 \\
\hline Transport & $1,602,111$ & $23,500,731$ & 14.67 & 5 & 15 & 5 & 15 \\
\hline ML_Laplace & 377,002 & $27,689,972$ & 73.45 & 26 & 74 & 26 & 74 \\
\hline RM07R & 381,689 & $37,464,962$ & 98.16 & 1 & 295 & 1 & 245 \\
\hline
\end{tabular}

All models are tested with 28 matrices chosen from the UFL matrix collection [63]. The properties of these matrices are presented in Table 2. The evaluated models are tested on a BlueGene/Q system with varying number of processors $K \in\{256$, 512, 1024, 2048, 4096, 8192\}. A node on this system consists of 16 cores (single PowerPC A2 processor) with $1.6 \mathrm{GHz}$ clock frequency and 16 GB memory. The nodes are connected by a 5D torus chip-to-chip network. We only consider the case of strong scaling.

For the pre-communication stages of $\mathrm{CKBD}+\mathrm{CHG}$ and JGD+CHG, we opted not to apply the communication hypergraph model since the partitioning corresponding to this stage leads to a number of very small communication hypergraphs in which the number of communication tasks (that is, vertices) and the number of messages per processor are very low. Hence, utilizing a dedicated tool to partition these hypergraphs often does not pay off. Instead, the simple aforementioned heuristic is able to obtain comparable partition qualities in a shorter amount of time. Hence, the pre-communication stages (expand tasks) of $\mathrm{CKBD}+\mathrm{BP}$ and $\mathrm{CKBD}+\mathrm{CHG}$ models, and JGD+BP and JGD+CHG models have the same quantities for the statistics presented in Section 4.1. However, for the post-communication stage, the respective quantities drastically differ in these models as the benefits of using the communication hypergraph model are more apparent.

\subsection{Bandwidth and latency costs of partitioning models}

Table 3 displays the metrics related to latency and bandwidth costs for the evaluated models. The metrics related to latency are highlighted under "Number of messages" columns and the metrics related to bandwidth are highlighted under "Communication volume" columns. The statistics for both total and maximum metrics are presented. The columns "Expand" and "Fold" in the table indicate the results obtained in the pre- and post-communication stages of 2D models, respectively. Recall that the $1 \mathrm{D}$ models $(1 \mathrm{D}+\mathrm{BP}$ and $1 \mathrm{D}+\mathrm{CHG}$ ) have a single communication stage, which is the pre-communication stage in our case. The values are averaged over 20 test matrices separately for each $K$. The communication volume statistics are in terms of words. Note that the total/maximum number of messages and maximum volume of communication of CKBD+BP and $\mathrm{CKBD}+\mathrm{CHG}$, and JGD+BP and JGD+CHG are the same since they use the same heuristic to distribute expand tasks. Total communication volume of two successive $K$ values (512 and 1024, 2048 and 4096, etc.) are the same for the models that are based on checkerboard and jagged partitioning since these models have the same number of processor columns in the corresponding processor mesh. For instance, at $K=512$ and $K=1024$, the processor meshes are of sizes $32 \times 16$ and 32 $\times 32$, respectively. We also present the average speedup values obtained by models to give an idea about the efficiency. A more detailed and accurate discussion with performance profiles and speedup curves can be found in the next section. 
Table 3

Average communication requirements and speedups.

\begin{tabular}{|c|c|c|c|c|c|c|c|c|c|c|c|c|c|c|}
\hline \multirow[b]{3}{*}{ K } & \multirow[b]{3}{*}{ Model } & \multicolumn{6}{|c|}{ Number of messages } & \multicolumn{6}{|c|}{ Communication volume } & \multirow[b]{3}{*}{ Speedup } \\
\hline & & \multicolumn{3}{|l|}{ Total } & \multicolumn{3}{|c|}{ Maximum } & \multicolumn{3}{|l|}{ Total } & \multicolumn{3}{|c|}{ Maximum } & \\
\hline & & Expand & Fold & Sum & Expand & Fold & Sum & Expand & Fold & Sum & Expand & Fold & Sum & \\
\hline \multirow{7}{*}{256} & $1 \mathrm{D}+\mathrm{BP}$ & 2464 & - & 2464 & 17.9 & - & 17.9 & 164470 & - & 164470 & 728 & - & 728 & 143 \\
\hline & $1 \mathrm{D}+\mathrm{CHG}$ & 1640 & - & 1640 & 13.5 & - & 13.5 & 245514 & - & 245514 & 1234 & - & 1234 & 148 \\
\hline & CKBD+BP & 414 & 1728 & 2141 & 4.8 & 6.6 & 11.4 & 38476 & 145554 & 184029 & 367 & 759 & 1125 & 142 \\
\hline & $\mathrm{CKBD}+\mathrm{CHG}$ & 414 & 1423 & 1836 & 4.8 & 5.8 & 10.5 & 38476 & 214657 & 253133 & 367 & 1176 & 1542 & 138 \\
\hline & JGD+BP & 850 & 1266 & 2117 & 10.0 & 4.9 & 14.9 & 38476 & 112712 & 151188 & 311 & 599 & 910 & 158 \\
\hline & $\mathrm{JGD}+\mathrm{CHG}$ & 850 & 1131 & 1982 & 10.0 & 5.0 & 15.0 & 38476 & 165081 & 203557 & 311 & 959 & 1270 & 153 \\
\hline & $\mathrm{FG}+\mathrm{BP}$ & 1959 & 1751 & 3710 & 15.5 & 6.8 & 22.3 & 107993 & 48359 & 156352 & 546 & 275 & 821 & 150 \\
\hline \multirow{10}{*}{512} & $\mathrm{FG}+\mathrm{CHG}$ & 1513 & 1257 & 2770 & 12.5 & 4.6 & 17.1 & 173449 & 74672 & 248121 & 847 & 377 & 1223 & 150 \\
\hline & $1 \mathrm{D}+\mathrm{BP}$ & 5683 & - & 5683 & 21.6 & - & 21.6 & 226127 & - & 226127 & 513 & - & 513 & 236 \\
\hline & $1 \mathrm{D}+\mathrm{CHG}$ & 3496 & _- & 3496 & 15.5 & _- & 15.5 & 327581 & _- & 327581 & 829 & _- & 829 & 231 \\
\hline & CKBD+BP & 1019 & 3590 & 4608 & 6.4 & 6.8 & 13.1 & 57434 & 195114 & 252548 & 264 & 530 & 794 & 225 \\
\hline & $\mathrm{CKBD}+\mathrm{CHG}$ & 1019 & 2885 & 3904 & 6.4 & 6.6 & 13.0 & 57434 & 280548 & 337981 & 264 & 804 & 1067 & 220 \\
\hline & JGD+BP & 2088 & 2604 & 4692 & 11.7 & 5.6 & 17.3 & 57434 & 147488 & 204922 & 220 & 405 & 626 & 247 \\
\hline & $\mathrm{JGD}+\mathrm{CHG}$ & 2088 & 2267 & 4355 & 11.7 & 4.5 & 16.2 & 57434 & 212002 & 269436 & 220 & 609 & 829 & 238 \\
\hline & $\mathrm{FG}+\mathrm{BP}$ & 4261 & 3784 & 8045 & 17.7 & 8.0 & 25.7 & 144968 & 70492 & 215460 & 374 & 205 & 580 & 228 \\
\hline & $\mathrm{FG}+\mathrm{CHG}$ & 3165 & 2622 & 5787 & 13.8 & 4.8 & 18.6 & 228987 & 106423 & 335410 & 566 & 273 & 839 & 229 \\
\hline & $1 \mathrm{D}+\mathrm{BP}$ & 13201 & - & 13201 & 27.0 & - & 27.0 & 314109 & - & 314109 & 359 & - & 359 & 294 \\
\hline \multirow{5}{*}{1024} & $1 \mathrm{D}+\mathrm{CHG}$ & 7451 & - & 7451 & 16.9 & - & 16.9 & 441340 & - & 441340 & 568 & - & 568 & 343 \\
\hline & $\mathrm{CKBD}+\mathrm{BP}$ & 1587 & 9624 & 11211 & 5.9 & 9.1 & 15.0 & 57434 & 288909 & 346343 & 167 & 399 & 567 & 320 \\
\hline & $\mathrm{CKBD}+\mathrm{CHG}$ & 1587 & 6897 & 8484 & 5.9 & 7.1 & 13.0 & 57434 & 404550 & 461984 & 167 & 534 & 701 & 320 \\
\hline & $\mathrm{JGD}+\mathrm{BP}$ & 3571 & 6775 & 10346 & 11.7 & 7.4 & 19.1 & 57434 & 223665 & 281099 & 138 & 314 & 452 & 354 \\
\hline & $\mathrm{JGD}+\mathrm{CHG}$ & 3571 & 5266 & 8837 & 11.7 & 5.2 & 16.9 & 57434 & 314303 & 371737 & 138 & 430 & 568 & 341 \\
\hline \multirow{10}{*}{2048} & $\mathrm{FG}+\mathrm{BP}$ & 9286 & 8141 & 17427 & 20.6 & 8.5 & 29.1 & 192165 & 103683 & 295848 & 252 & 153 & 405 & 318 \\
\hline & $\mathrm{FG}+\mathrm{CHG}$ & 6537 & 5420 & 11957 & 15.1 & 6.1 & 21.3 & 297236 & 152370 & 449606 & 376 & 201 & 577 & 327 \\
\hline & $1 \mathrm{D}+\mathrm{BP}$ & 30651 & - & 30651 & 30.9 & - & 30.9 & 437009 & - & 437009 & 256 & - & 256 & 355 \\
\hline & $1 \mathrm{D}+\mathrm{CHG}$ & 16028 & - & 16028 & 19.0 & - & 19.0 & 591207 & - & 591207 & 389 & - & 389 & 450 \\
\hline & CKBD+BP & 3885 & 21008 & 24892 & 7.3 & 9.4 & 16.7 & 82863 & 395862 & 478725 & 116 & 277 & 393 & 421 \\
\hline & $\mathrm{CKBD}+\mathrm{CHG}$ & 3885 & 14275 & 18159 & 7.3 & 8.5 & 15.8 & 82863 & 533056 & 615920 & 116 & 362 & 478 & 429 \\
\hline & $\mathrm{JGD}+\mathrm{BP}$ & 8301 & 14621 & 22921 & 14.1 & 7.4 & 21.5 & 82863 & 297989 & 380852 & 99 & 214 & 313 & 468 \\
\hline & $\mathrm{JGD}+\mathrm{CHG}$ & 8301 & 10833 & 19134 & 14.1 & 5.6 & 19.6 & 82863 & 404389 & 487252 & 99 & 284 & 383 & 457 \\
\hline & $\mathrm{FG}+\mathrm{BP}$ & 20050 & 17669 & 37719 & 22.3 & 10.3 & 32.6 & 251398 & 154339 & 405737 & 171 & 116 & 287 & 412 \\
\hline & $\mathrm{FG}+\mathrm{CHG}$ & 13494 & 11272 & 24766 & 16.3 & 8.2 & 24.5 & 379611 & 221040 & 600651 & 243 & 152 & 395 & 440 \\
\hline \multirow{7}{*}{4096} & $1 \mathrm{D}+\mathrm{BP}$ & 71313 & - & 71313 & 37.0 & - & 37.0 & 615662 & - & 615662 & 185 & - & 185 & 372 \\
\hline & $1 \mathrm{D}+\mathrm{CHG}$ & 36563 & - & 36563 & 21.8 & - & 21.8 & 810012 & - & 810012 & 267 & - & 267 & 530 \\
\hline & $\mathrm{CKBD}+\mathrm{BP}$ & 6122 & 55186 & 61308 & 6.7 & 13.5 & 20.3 & 82863 & 579257 & 662121 & 72 & 209 & 280 & 511 \\
\hline & $\mathrm{CKBD}+\mathrm{CHG}$ & 6122 & 32230 & 38352 & 6.7 & 8.7 & 15.4 & 82863 & 747663 & 830526 & 72 & 252 & 324 & 556 \\
\hline & $\mathrm{JGD}+\mathrm{BP}$ & 13440 & 38102 & 51543 & 12.9 & 10.4 & 23.2 & 82863 & 449614 & 532477 & 62 & 163 & 225 & 584 \\
\hline & $\mathrm{JGD}+\mathrm{CHG}$ & 13440 & 24711 & 38151 & 12.9 & 6.7 & 19.5 & 82863 & 584442 & 667306 & 62 & 203 & 264 & 586 \\
\hline & $\mathrm{FG}+\mathrm{BP}$ & 43084 & 38239 & 81323 & 24.8 & 11.0 & 35.7 & 326646 & 228666 & 555312 & 114 & 87 & 201 & 516 \\
\hline \multirow{9}{*}{8192} & $\mathrm{FG}+\mathrm{CHG}$ & 28683 & 24606 & 53289 & 18.0 & 8.8 & 26.7 & 486581 & 323623 & 810204 & 160 & 117 & 278 & 554 \\
\hline & 1D+BP & 160507 & - & 160507 & 42.9 & - & 42.9 & 871090 & - & 871090 & 137 & - & 137 & 392 \\
\hline & $1 \mathrm{D}+\mathrm{CHG}$ & 81592 & - & 81592 & 26.0 & - & 26.0 & 1040719 & - & 1040719 & 175 & - & 175 & 560 \\
\hline & $\mathrm{CKBD}+\mathrm{BP}$ & 14589 & 125660 & 140249 & 7.9 & 15.7 & 23.6 & 115948 & 814437 & 930384 & 49 & 149 & 198 & 593 \\
\hline & $\mathrm{CKBD}+\mathrm{CHG}$ & 14589 & 70942 & 85531 & 7.9 & 10.7 & 18.6 & 115948 & 1003817 & 1119764 & 49 & 171 & 220 & 667 \\
\hline & $\mathrm{JGD}+\mathrm{BP}$ & 28838 & 85171 & 114009 & 13.9 & 11.4 & 25.2 & 115948 & 618294 & 734242 & 43 & 114 & 157 & 683 \\
\hline & $\mathrm{JGD}+\mathrm{CHG}$ & 28838 & 52882 & 81720 & 13.9 & 6.5 & 20.3 & 115948 & 769539 & 885486 & 43 & 137 & 180 & 701 \\
\hline & $\mathrm{FG}+\mathrm{BP}$ & 90483 & 81319 & 171801 & 26.3 & 13.3 & 39.6 & 430725 & 327017 & 757741 & 77 & 65 & 142 & 591 \\
\hline & $\mathrm{FG}+\mathrm{CHG}$ & 59692 & 52380 & 112072 & 19.8 & 10.1 & 29.9 & 572597 & 432085 & 1004682 & 99 & 84 & 183 & 663 \\
\hline
\end{tabular}

The bold values in five columns total/maximum number of messages (Sum), total/maximum communication volume (Sum) and Speedup indicate the best values obtained by the respective model at a specific $K$.

The major factors that determine overall latency and bandwidth costs are the maximum number of messages and the maximum volume of communication handled by a single processor, respectively. As seen in Table 3, with increasing number of processors, the maximum number of messages increases sharply, whereas maximum volume decreases despite the increase in total volume. For instance, for 1D+BP, when $K$ increases from 256 to 8192 processors, the maximum number of messages increases from 17.9 to 42.9 , whereas maximum volume decreases from 728 to 137 words, on average. Hence, the latency overhead on average increases by a factor of 2.4, whereas the bandwidth overhead on average decreases by a factor of 5.3. Moreover, the total message count increases more sharply compared to total volume: 65.1 times versus 5.3 times. These figures imply that with increasing number of processors, latency costs steadily become more important than bandwidth costs in determining overall communication cost of parallel SpMV operations. Hence, reducing latency costs should pay off with improved scalability, as will be seen in the following section. Observe that similar arguments hold for other partitioning models as well. 
Table 4

Comparison of partitioning models with communication hypergraphs normalized with respect to their baseline counterparts averaged over all matrices for each $K$.

\begin{tabular}{|c|c|c|c|c|c|c|c|c|c|c|c|c|c|}
\hline \multirow[b]{3}{*}{ K } & \multirow[b]{3}{*}{ Model } & \multicolumn{6}{|c|}{ Number of messages } & \multicolumn{6}{|c|}{ Communication volume } \\
\hline & & \multicolumn{3}{|l|}{ Total } & \multicolumn{3}{|c|}{ Maximum } & \multicolumn{3}{|l|}{ Total } & \multicolumn{3}{|c|}{ Maximum } \\
\hline & & Expand & Fold & Sum & Expand & Fold & Sum & Expand & Fold & Sum & Expand & Fold & Sum \\
\hline & $1 \mathrm{D}+\mathrm{CHG}$ & 0.76 & - & 0.76 & 0.85 & - & 0.85 & 1.51 & - & 1.51 & 1.66 & - & 1.66 \\
\hline \multirow[t]{4}{*}{256} & $\mathrm{CKBD}+\mathrm{CHG}$ & 1.00 & 0.86 & 0.89 & 1.00 & 1.01 & 0.98 & 1.00 & 1.49 & 1.41 & 1.00 & 1.54 & 1.38 \\
\hline & $\mathrm{JGD}+\mathrm{CHG}$ & 1.00 & 0.95 & 0.98 & 1.00 & 1.21 & 1.09 & 1.00 & 1.45 & 1.35 & 1.00 & 1.53 & 1.36 \\
\hline & $\mathrm{FG}+\mathrm{CHG}$ & 0.87 & 0.85 & 0.86 & 0.96 & 0.81 & 0.90 & 1.56 & 1.51 & 1.56 & 1.53 & 1.26 & 1.47 \\
\hline & $1 \mathrm{D}+\mathrm{CHG}$ & 0.72 & - & 0.72 & 0.84 & - & 0.84 & 1.48 & - & 1.48 & 1.62 & - & 1.62 \\
\hline \multirow[t]{4}{*}{512} & $\mathrm{CKBD}+\mathrm{CHG}$ & 1.00 & 0.85 & 0.88 & 1.00 & 1.41 & 1.08 & 1.00 & 1.46 & 1.37 & 1.00 & 1.52 & 1.35 \\
\hline & $\mathrm{JGD+CHG}$ & 1.00 & 0.92 & 0.96 & 1.00 & 0.97 & 0.97 & 1.00 & 1.42 & 1.32 & 1.00 & 1.50 & 1.33 \\
\hline & $\mathrm{FG}+\mathrm{CHG}$ & 0.85 & 0.84 & 0.84 & 0.94 & 0.80 & 0.88 & 1.55 & 1.50 & 1.54 & 1.52 & 1.23 & 1.44 \\
\hline & $1 \mathrm{D}+\mathrm{CHG}$ & 0.67 & - & 0.67 & 0.77 & - & 0.77 & 1.44 & - & 1.44 & 1.61 & - & 1.61 \\
\hline \multirow[t]{4}{*}{1024} & $\mathrm{CKBD}+\mathrm{CHG}$ & 1.00 & 0.78 & 0.81 & 1.00 & 1.03 & 0.97 & 1.00 & 1.44 & 1.38 & 1.00 & 1.37 & 1.27 \\
\hline & JGD+CHG & 1.00 & 0.86 & 0.91 & 1.00 & 0.88 & 0.94 & 1.00 & 1.41 & 1.34 & 1.00 & 1.42 & 1.29 \\
\hline & $\mathrm{FG}+\mathrm{CHG}$ & 0.81 & 0.81 & 0.80 & 0.86 & 1.37 & 0.87 & 1.53 & 1.48 & 1.51 & 1.51 & 1.24 & 1.43 \\
\hline & $1 \mathrm{D}+\mathrm{CHG}$ & 0.64 & - & 0.64 & 0.74 & - & 0.74 & 1.40 & - & 1.40 & 1.57 & - & 1.57 \\
\hline \multirow[t]{4}{*}{2048} & $\mathrm{CKBD}+\mathrm{CHG}$ & 1.00 & 0.74 & 0.77 & 1.00 & 1.02 & 0.99 & 1.00 & 1.39 & 1.33 & 1.00 & 1.33 & 1.23 \\
\hline & JGD+CHG & 1.00 & 0.82 & 0.87 & 1.00 & 0.94 & 0.95 & 1.00 & 1.37 & 1.30 & 1.00 & 1.37 & 1.25 \\
\hline & $\mathrm{FG}+\mathrm{CHG}$ & 0.77 & 0.77 & 0.77 & 0.82 & 0.96 & 0.84 & 1.51 & 1.45 & 1.49 & 1.46 & 1.32 & 1.41 \\
\hline & 1D+CHG & 0.65 & - & 0.65 & 0.68 & - & 0.68 & 1.39 & - & 1.39 & 1.54 & - & 1.54 \\
\hline \multirow[t]{4}{*}{4096} & $\mathrm{CKBD}+\mathrm{CHG}$ & 1.00 & 0.66 & 0.69 & 1.00 & 0.77 & 0.84 & 1.00 & 1.35 & 1.31 & 1.00 & 1.27 & 1.19 \\
\hline & $\mathrm{JGD+CHG}$ & 1.00 & 0.74 & 0.80 & 1.00 & 0.82 & 0.90 & 1.00 & 1.33 & 1.29 & 1.00 & 1.33 & 1.23 \\
\hline & $\mathrm{FG}+\mathrm{CHG}$ & 0.77 & 0.78 & 0.77 & 0.82 & 0.85 & 0.81 & 1.50 & 1.46 & 1.49 & 1.45 & 1.38 & 1.42 \\
\hline & $1 \mathrm{D}+\mathrm{CHG}$ & 0.63 & - & 0.63 & 0.69 & - & 0.69 & 1.35 & - & 1.35 & 1.47 & - & 1.47 \\
\hline \multirow[t]{3}{*}{8192} & $\mathrm{CKBD}+\mathrm{CHG}$ & 1.00 & 0.64 & 0.67 & 1.00 & 0.79 & 0.84 & 1.00 & 1.30 & 1.26 & 1.00 & 1.23 & 1.16 \\
\hline & JGD+CHG & 1.00 & 0.69 & 0.76 & 1.00 & 0.70 & 0.85 & 1.00 & 1.29 & 1.25 & 1.00 & 1.30 & 1.21 \\
\hline & $\mathrm{FG}+\mathrm{CHG}$ & 0.76 & 0.76 & 0.76 & 0.82 & 0.75 & 0.78 & 1.47 & 1.42 & 1.45 & 1.44 & 1.40 & 1.42 \\
\hline
\end{tabular}

If we compare the partitioning models that do not use the communication hypergraph model among themselves (i.e., $1 D+B P, C K B D+B P, J G D+B P$ and $F G+B P$ ) in terms of total communication volume, we see from Table 3 that JGD+BP obtains the best results, whereas $C K B D+B P$ obtains the worst results. FG+BP is expected to achieve the best results in this metric since it offers the highest flexibility by performing the partitioning on a nonzero basis - the finest granularity available. However, the reason why JGD+BP achieves slightly better results than FG+BP in this metric is related not to models themselves but to the shortcomings of recursive bisectioning used in partitioning. The shortcomings of recursive bipartitioning are well known for high partitioning values [64,65]. For example at $K=4096, \mathrm{FG}+\mathrm{BP}$ directly partitions the input matrix into 4096 parts whereas JGD+BP first partitions it into 64 parts, and for each of these parts, it partitions them into 64 parts again to obtain a 4096-way partition. Hence, by using smaller partition values compared to FG+BP, JGD+BP is relatively able to mitigate the drawbacks of recursive bisection. The poor performance of CKBD+BP in this metric is due to the use of multi-constraint partitioning. This limits the search space drastically, where the higher the number of constraints, the harder it is to get good quality partitions as the search space narrows down with increasing number of constraints. However, this is a tradeoff for CKBD+BP as it often achieves good results in total message count, which are comparable to those of JGD+BP at lower processor counts. At higher processor counts, like 4096 and 8192, JGD+BP achieves better results in total message count. This is again because the high number of constraints at high values of $K$ leads to poor total volume in $\mathrm{CKBD}+\mathrm{BP}$, which in turn affects the total message count as a side effect by causing an increase. As expected, the smallest maximum number of messages is obtained by CKBD+BP as it bounds the communication to specific rows and columns of the processor mesh in both stages of communication. $\mathrm{FG}+\mathrm{BP}$ is often the worst in terms of total and maximum number of messages because it causes increases in these metrics in order to reduce total volume.

To aid the assessment of benefits of the communication hypergraph, we present Table 4 . In this table, each latencyimproved (CHG-enhanced) model's performance metrics are normalized with respect to those of their baselines. In other words, the results of $1 \mathrm{D}+\mathrm{CHG}$ are normalized with respect to those of $1 \mathrm{D}+\mathrm{BP}$, the results of $\mathrm{CKBD}+\mathrm{CHG}$ are normalized with respect to those of $\mathrm{CKBD+BP}$, etc. The normalization is performed on a matrix basis and the averages of these normalized values over 28 matrices are given separately for each $K$. As seen from the table, CHG-enhanced models improve the total message count drastically, as minimizing this metric is one of the main objectives in these models. For example at 2048 processors, $1 \mathrm{D}+\mathrm{CHG}$ achieves $36 \%$ improvement over $1 \mathrm{D}+\mathrm{BP}, \mathrm{CKBD}+\mathrm{CHG}$ achieves $23 \%$ improvement over CKBD+BP, JGD+CHG achieves $13 \%$ improvement over JGD+BP, and FG+CHG achieves $23 \%$ improvement over FG+BP. However, this comes at the cost of increased total volume. Again at 2048 processors, $1 \mathrm{D}+\mathrm{CHG}$ increases the total volume by $40 \%$, CKBD $+\mathrm{CHG}$ by $33 \%$, $\mathrm{JGD}+\mathrm{CHG}$ by $30 \%$, and $\mathrm{FG}+\mathrm{CHG}$ by $49 \%$. The crucial observation, however, is that the message count improvements of partitioning models that rely on the communication hypergraph model tend to increase with increasing number of processors. For example, $1 \mathrm{D}+\mathrm{CHG}$ achieves a $24 \%$ improvement over $1 \mathrm{D}+\mathrm{BP}$ at $K=256$ in total message count and this improvement becomes $37 \%$ at $K=8192$. This improvement increases from $11 \%$ to $33 \%, 2 \%$ to $24 \%$, and $14 \%$ to $24 \%$ for CKBD+CHG, JGD+CHG 
Table 5

Average partitioning times (sequential, in seconds).

\begin{tabular}{lllll}
\hline & 1D+BP & CKBD+BP & JGD+BP & FG+BP \\
\hline & 32.43 & 33.79 & 21.68 & 114.22 \\
CHG & 1.68 & 0.98 & 1.13 & 1.98 \\
\hline
\end{tabular}

and $\mathrm{FG}+\mathrm{CHG}$, respectively. This implies that the benefits obtained using the communication hypergraph model are more prominent at higher processor counts. Note that for $\mathrm{CKBD}+\mathrm{CHG}$ and $\mathrm{JGD}+\mathrm{CHG}$, normalized total message average is closer to fold message average rather than expand message average, which is also the case for normalized average volume. This is because the message count and communication volume in the post-communication stage are much higher than the precommunication stage in these models. This is also where the communication hypergraph model is expected to perform well.

The models that use the communication hypergraph model improve the maximum number of messages as well. This is a consequence of the reduction in total message count. In Table 3, if we compare partitioning models in this metric, it can be seen that CKBD+CHG obtains the best results which is usually followed by JGD+CHG. For example, at 8192 processors on average, the maximum number of messages handled by a single processor for CKBD+CHG is only 18.6 and for JGD+CHG it is only 20.3. These values are followed by CKBD+BP with 23.6 and JGD+BP with 25.2. When we examine the other important metric the maximum volume in Table 4, it is seen that the models that rely on the communication hypergraph model close the gap with their baseline counterparts with increasing $K$. For instance, when $K$ increases from 256 to 8192 processors, the increase in maximum volume incurred by the use of the communication hypergraph model decreases from $66 \%, 38 \%, 36 \%$ and $47 \%$ to $47 \%, 16 \%, 21 \%$ and $42 \%$ in $1 \mathrm{D}+\mathrm{CHG}, \mathrm{CKBD}+\mathrm{CHG}, \mathrm{JGD}+\mathrm{CHG}$ and $\mathrm{FG}+\mathrm{CHG}$, respectively, compared to their baseline counterparts. This is an important benefit of the communication hypergraph model since it strives for balancing volume.

The sequential partitioning times of the evaluated models are given in Table 5 averaged over all matrices and $K$ values. The CHG times (indicated via CHG row) include only the partitioning times of the communication hypergraphs formed for the respective model. As expected, the fine-grain model has the highest partitioning time as the hypergraphs formed in this model are typically larger. The partitioning times of the communication hypergraphs are quite low compared to the respective original partitionings since they are small as they contain only the vertices that correspond to the vector elements that necessitate communication. Note that $\mathrm{CKBD}+\mathrm{CHG}$, JGD+CHG and FG+CHG form a number of communication hypergraphs that can independently be partitioned, hence the partitioning of them can easily be parallelized. A more healthy comparison of partitioning overhead for 2D models can be found in [49].

\subsection{Speedup analysis}

For a detailed comparison of the partitioning models in terms of parallel solver running times/speedups, we present the runtime performance profiles in Fig. 8. Performance profiles provide a better understanding of the characteristics of the compared models as they capture the relative performance of the compared models more accurately [66]. A point $x$, $y$ in a profile reads as the respective model is within a $x$ factor of the best result in a $y$ fraction of the test instances. In other words, the closer the performance profile of a scheme to the $y$-axis, the better it is. A test instance in our case is the parallel solver running time obtained for a specific matrix and $K$. We compare the performances of partitioning models for all $K$ values in Fig. 8a and for $K \in$ 4096, 8192 in Fig. 8b. The former contains 168 instances and the latter contains 56 instances.

When we compare the models considering all $K$ values in Fig. 8a, JGD+BP is clearly the best performing model followed by JGD+CHG. JGD+BP obtains the best results for more than $40 \%$ of the test cases and exhibits very good performance for a very large fraction of the test cases. These two models are followed by two models that use the communication hypergraph: $1 \mathrm{D}+\mathrm{CHG}$ and $\mathrm{FG}+\mathrm{CHG}$. Except the jagged model, applying the communication hypergraph seems to improve performance of the partitioning models as $1 \mathrm{D}+\mathrm{CHG}, \mathrm{CKBD}+\mathrm{CHG}$ and $\mathrm{FG}+\mathrm{CHG}$ perform better than $1 \mathrm{D}+\mathrm{BP}, \mathrm{CKBD}+\mathrm{BP}$ and $\mathrm{FG}+\mathrm{BP}$, respectively. $1 \mathrm{D}+\mathrm{BP}$ obtains the worst results, proving itself to be not a viable partitioning model compared to the $2 \mathrm{D}$ models as long as the communication hypergraph is not used for it.

Fig. $8 \mathrm{~b}$ is presented to better assess the benefits of using the communication hypergraph model. As discussed, latency gets more important with increasing $K$ and it is expected that the models using the communication hypergraph model should be performing better as $K$ increases. If we consider the performances of partitioning models at only 4096 and 8192 processors, it can be seen from the figure that the models that use the communication hypergraph improve the performance much more compared to the case when all $K$ values are considered. In other words, for example, if we compare CKBD+BP and $\mathrm{CKBD}+\mathrm{CHG}$ in Fig. $8 \mathrm{a}$ and Fig. $8 \mathrm{~b}$ the performance difference between them increases in favor of CKBD+CHG in Fig. 8b. This can be observed for all partitioning models, i.e., by comparing $1 \mathrm{D}+\mathrm{BP}$ and $1 \mathrm{D}+\mathrm{CHG}, \mathrm{CKBD}+\mathrm{BP}$ and $\mathrm{CKBD}+\mathrm{CHG}, \mathrm{JGD}+\mathrm{BP}$ and JGD+CHG, FG+BP and FG+CHG in Fig. 8a and Fig. 8b. This is also validated as JGD+CHG can be said to be the best performing model in Fig. 8b followed by JGD+BP. These two models are again followed by two models that use the communication hypergraph: $\mathrm{FG}+\mathrm{CHG}$ and $\mathrm{CKBD}+\mathrm{CHG}$. These figures show that the communication hypergraph proves to be a valuable method for achieving scalability. 


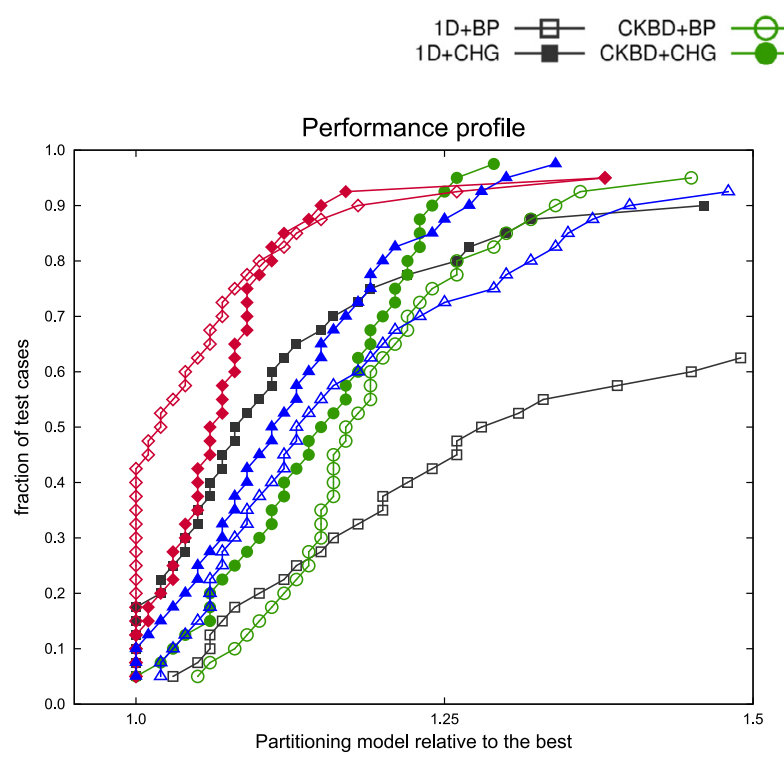

(a) $K \in\{256,512,1024,2048,4096,8192\}$.
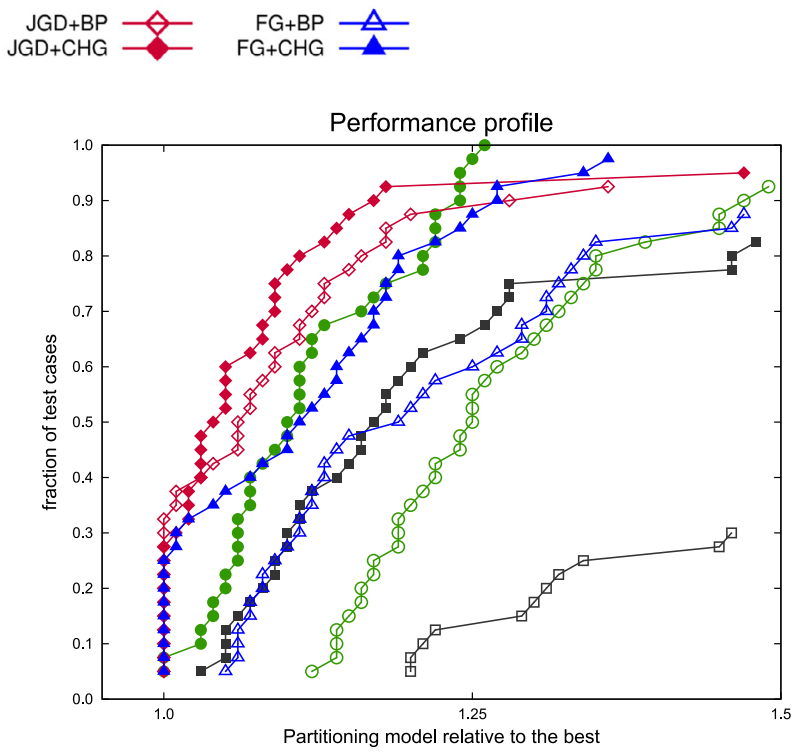

(b) $K \in\{4096,8192\}$.

Fig. 8. Runtime performance profiles of eight partitioning models.

We present the obtained speedup values of eight evaluated models in Fig. 9. Among 28 matrices, we present the speedups of 12 matrices here to keep the discussions simpler. The rest are given in Appendix G. The number of processors varies from 256 to 8192. The experiments are performed with the CGNR solver which is implemented via the PETSc toolkit.

As seen from the speedup curves, the models that adopt the communication hypergraph model often exhibit better scalability compared to their baseline counterparts. Moreover, the difference gets more prominent with increasing number of processors as latency becomes the determining factor for performance. When we compare $1 \mathrm{D}+\mathrm{BP}$ with $1 \mathrm{D}+\mathrm{CHG}, 1 \mathrm{D}+\mathrm{CHG}$ achieves superior scalability in all matrices. In 2D models, applying the communication hypergraph model usually improves scalability. For example, in matrices circuit5M_dc, CoupCons3D, fem_hifreq_circuit, Freescale1, memchip, ML_Laplace, rajat31 and RM07R, the partitioning models CKBD+CHG, JGD+CHG and FG+CHG improve performance of their baseline counterparts $C K B D+B P, J G D+B P$ and $F G+B P$, respectively. In the remaining matrices, latency-improved versions of 2D models either achieve close or slightly worse performance. This is mainly due to the fact that in these matrices, the latency costs obtained in the initial partitionings are already very low due to the characteristics of these matrices and further trying to improve them does not pay off since, at the other hand, the bandwidth costs are increased.

In terms of speedup values, it can be said that $2 \mathrm{D}$ models generally exhibit better scalability than $1 \mathrm{D}$ models. In most of the matrices, the best of $2 \mathrm{D}$ models exhibits better scalability than the best of $1 \mathrm{D}$ models, by obtaining lower runtime results. With increasing number of processors, this difference becomes more obvious. This can be attributed to the fact that 2D models have more flexibility in partitioning, which leads them to optimize communication objectives better.

Among all models, the performance of JGD+BP and JGD+CHG is especially worth to note. On average, these two models achieve quite good performance in terms of speedup. In matrices atmosmodl, Chevron4 and kim2, JGD+BP obtains better speedup values. The communication costs of these matrices are largely determined by bandwidth costs rather than by latency costs. From this point of view, FG+BP might be expected to achieve the best results. However, FG+BP usually causes high latency costs, even in the case of these matrices which are not latency bound. JGD+BP, on the other hand, obtains slightly worse bandwidth costs while drastically improving latency costs compared to $\mathrm{FG}+\mathrm{BP}$, finding a balance between $\mathrm{FG}+\mathrm{BP}$ and $\mathrm{CKBD}+\mathrm{BP}$, hence performing best in these matrices.

In the remaining nine matrices, the latency-improved versions of $2 \mathrm{D}$ models obtain better scalability. The JGD+CHG model is almost always among the two best performing models. When comparing CKBD+CHG, JGD+CHG and FG+CHG, although $\mathrm{CKBD}+\mathrm{CHG}$ has the lowest maximum number of messages, it has the highest maximum volume, whereas JGD+CHG obtains slightly worse maximum number of messages compared to CKBD+CHG and has the lowest maximum volume among these three models. Hence it is able to strike a good balance between minimizing latency and bandwidth costs, which leads to better scalability. Although $\mathrm{FG}+\mathrm{CHG}$ has low bandwidth costs, its high latency costs cause it to perform relatively poorly among these models (for example for rajat31 matrix, the maximum number of messages of FG+CHG at 4096 processors is around hundreds).

A noteworthy case is seen for the cage13 matrix. This matrix is characterized with its very high latency cost. For example, at $K=8192$ the maximum number of messages is 345 for $1 \mathrm{D}+\mathrm{BP}$. In such matrices, bounding and reducing the 


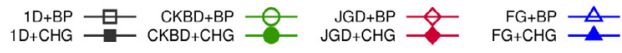
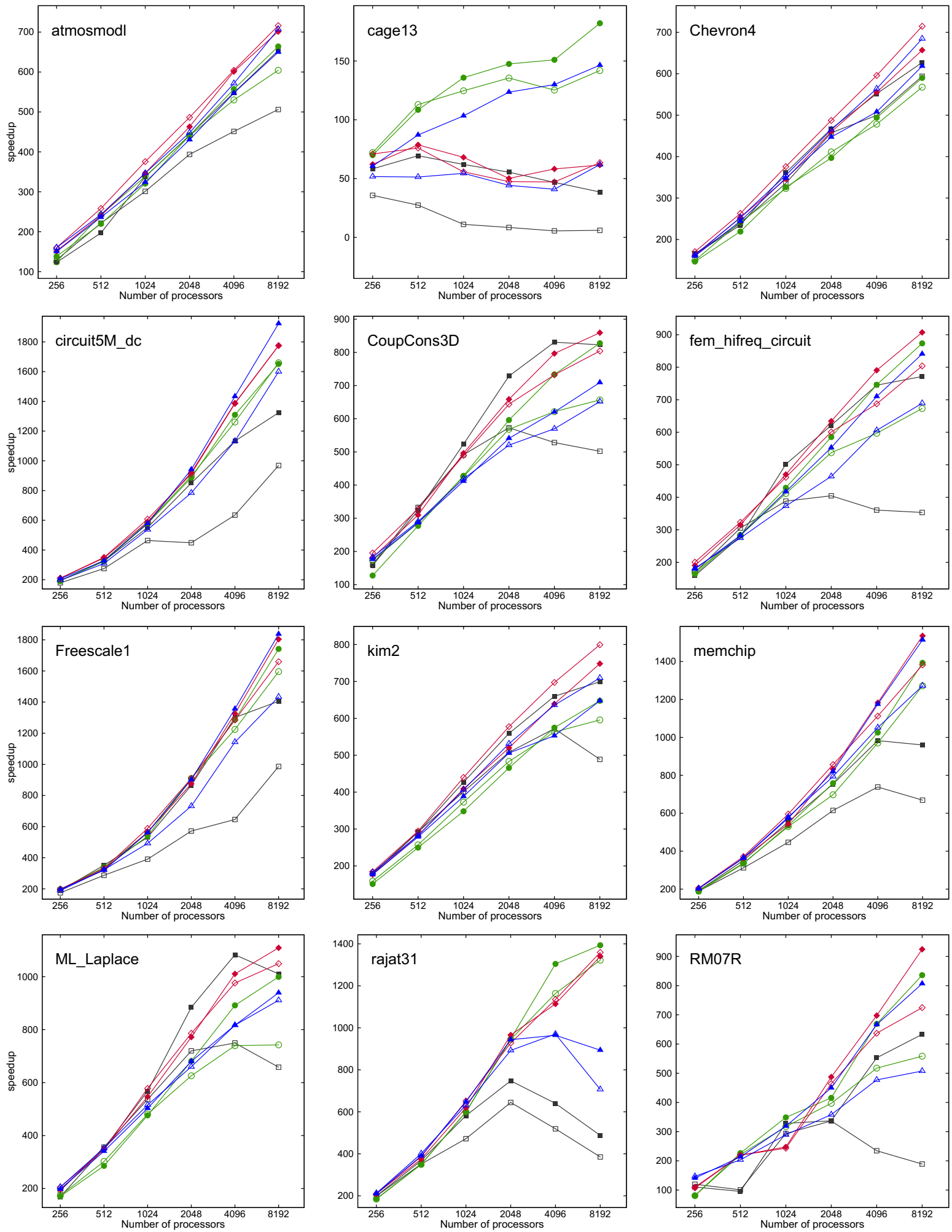

Fig. 9. Speedup curves. 
message count works better than by solely reducing it. As seen in Fig. 9, the two models that do so, CKBD+BP and CKBD+CHG, achieve better scalability.

Judging from performance profiles and speedup curves, we can safely recommend the use of the JGD+CHG model when latency costs prove vital in performance, and JGD+BP model when latency and bandwidth costs are comparable. Although other models may perform better for specific matrices in some cases, it can be said that JGD+BP and JGD+CHG will not perform too inferior even in these cases (for example, circuit5M_dc matrix).

\section{Conclusions}

This work focused on reducing latency costs of parallel sparse-matrix vector operations by proposing and utilizing several models based on 1D and 2D matrix partitioning. The latency costs are improved by using the communication hypergraph models, where the main motivation is to minimize the number of messages communicated in parallel operations. The described and tested models are realized with CGNE and CGNR solvers via the PETSc toolkit on a modern HPC system. We compared a total of eight partitioning models, scaling them up to $8 \mathrm{~K}$ processors.

The results of extensive experiments indicate that along with the bandwidth costs, latency costs should certainly be considered in order to achieve scalable performance. Solely addressing a single of them hurts scalability and leads to poor performance. Our findings indicate that among the partitioning models, the 2D jagged model and its latency-improved version obtain the most promising results. This superior performance is the result of obtaining a good balance between minimizing latency and bandwidth costs.

\section{Acknowledgements}

We acknowledge PRACE (Partnership for Advanced Computing In Europe) for awarding us access to resource Juqueen (Blue Gene/Q) based in Germany at Jülich Supercomputing Centre.

\section{Appendix A. Hypergraph partitioning}

A hypergraph $\mathcal{H}=(\mathcal{V}, \mathcal{N})$ consists of a set of vertices $\mathcal{V}$ and a set of nets $\mathcal{N}$ [67]. Each net $n_{j} \in \mathcal{N}$ connects a subset of vertices, which are referred to as pins of $n_{j}$. The set of nets that connect vertex $v_{i}$ is denoted by $\operatorname{Nets}\left(v_{i}\right)$. The degree of a vertex is equal to the number of nets that connect this vertex, i.e., $d_{i}=\left|\operatorname{Nets}\left(v_{i}\right)\right|$. A weight value $w_{i}$ is associated with each vertex $v_{i}$.

Given a hypergraph $\mathcal{H}=(\mathcal{V}, \mathcal{N}), \Pi=\left\{\mathcal{V}_{1}, \mathcal{V}_{2}, \ldots, \mathcal{V}_{K}\right\}$ is called a $K$-way partition of vertex set $\mathcal{V}$ if each part $V_{k}$ is nonempty, parts are pairwise disjoint and the union of $K$ parts is equal to $\mathcal{V}$. In $\Pi$, a net is said to connect a part if it connects at least one vertex in that part. The set of parts connected by a net $n_{j}$ is called its connectivity set and is denoted by $\Lambda\left(n_{j}\right)$. The connectivity $\lambda\left(n_{j}\right)=\left|\Lambda\left(n_{j}\right)\right|$ of $n_{j}$ is equal to the number of parts connected by this net. Net $n_{j}$ is said to be an internal net if it connects only one part $\left(\lambda\left(n_{j}\right)=1\right)$, and an external net if it connects more than one part $\left(\lambda\left(n_{j}\right)>1\right)$. In $\Pi$, the weight of a part is the sum of the weights of vertices in that part. In the hypergraph partitioning (HP) problem, the objective is to minimize the cutsize, which is defined as cutsize $(\Pi)=\sum_{n_{j} \in \mathcal{N}}\left(\lambda\left(n_{j}\right)-1\right)$. This objective function is known as the connectivity- 1 cutsize metric and is widely used in the scientific computing community [31,68,69]. The partitioning constraint is to satisfy a balance on part weights, $\left(W_{\max }-W_{\text {avg }}\right) / W_{\text {avg }} \leq \epsilon$, where $W_{\max }$ and $W_{\text {avg }}$ are the maximum and the average part weights, respectively, and $\epsilon$ is the user-defined imbalance ratio. The HP problem is known to be NP-hard [70]. Nonetheless, there exist successful HP tools such as PaToH [31], hMeTiS [60] and Mondriaan [33].

\section{Appendix B. Communication requirements of 1D partitioning}

In 1D partitioning, $n \times n$ matrix $A$ is partitioned either row wise or column wise. Assume that $A$ is permuted into a $K \times$ $K$ block structure as follows:

$$
A_{B L}=\left[\begin{array}{cccc}
A_{11} & A_{12} & \ldots & A_{1 K} \\
A_{21} & A_{22} & \ldots & A_{2 K} \\
\vdots & \vdots & \ddots & \vdots \\
A_{K 1} & A_{K 2} & \ldots & A_{K K}
\end{array}\right],
$$

where $K$ denotes the number of processors in the parallel system and the size of block $A_{k l}$ is $n_{k} \times n_{l}$. In row wise partitioning, processor $P_{k}$ is responsible for the $k$ th row $\left[A_{k 1} \ldots A_{k K}\right]$ of size $n_{k} \times n$. In column wise partitioning, processor $P_{k}$ is responsible for the $k$ th column block $\left[A_{1 k}^{T} \ldots A_{K k}^{T}\right]^{T}$ of size $n \times n_{k}$. Throughout this section, without loss of generality, we assume a row wise partition of $A$.

The vectors in an iterative solver should be partitioned conformally in order to avoid redundant communication during linear vector operations. For example, in the conjugate gradient solver, all vectors are partitioned conformally. In some solvers, we can utilize distinct vector partitions that separately apply to certain vectors. For example in CGNE and CGNR, it is possible to utilize two distinct partitions on the vectors. This enables utilization of a nonsymmetric partition for the coefficient matrix (see Appendix E for the details). The main motivation for adopting a nonsymmetric partition is that instead 


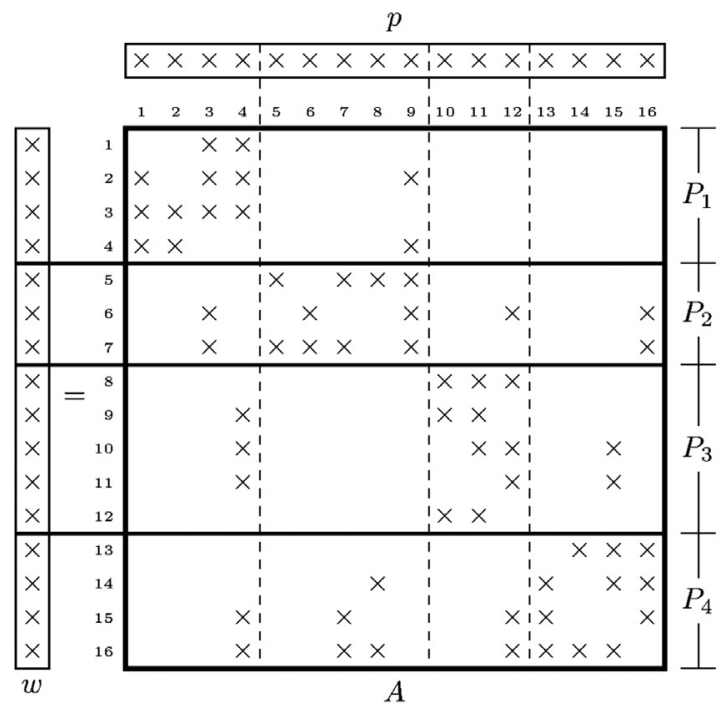

(a) Row-parallel $w=A p$.

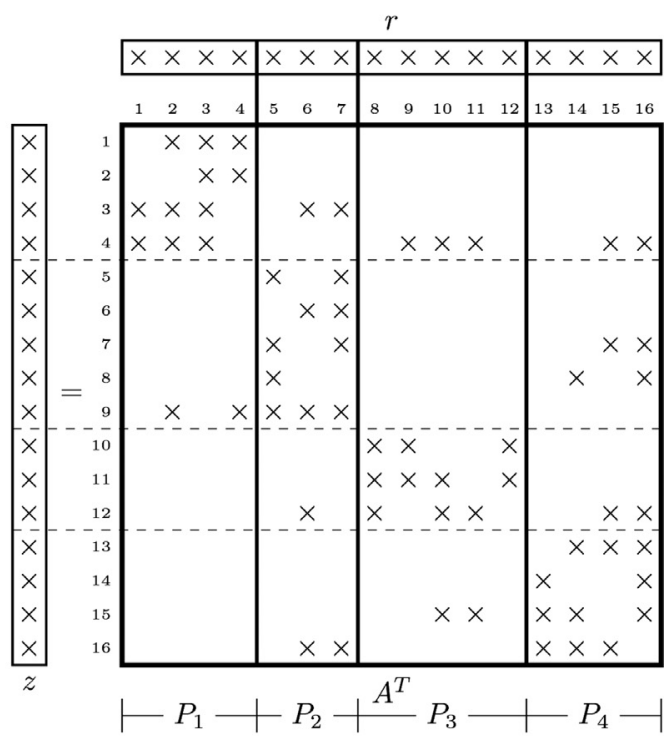

(b) Column-parallel $z=A^{T} r$.

Fig. B10. Row-parallel matrix-vector and column-parallel matrix-transpose-vector multiplication.

of enforcing the same partition on all vectors in the solver, we have more freedom by using a different partition on each distinct vector space - which accommodates more potential for reducing communication overheads in parallelization.

In a parallel iterative solver, inner product operations necessitate global collective communications whereas matrix-vector or matrix-transpose-vector multiplications necessitate P2P communications. Consider parallel $w=A p$ and $z=A^{T} r$ multiplies. An example for these operations is illustrated in Fig. B.10 for $K=4$ processors. Without loss of generality, assume that $P_{k}$ is responsible for the kth row stripe of $A$, and thus the kth column stripe of $A^{T}$. Note that a row wise partition on $A$ induces a column wise partition on $A^{T}$ (Appendix E). Here, $w=A p$ is performed with the row-parallel algorithm while $z=A^{T} r$ is performed with the column-parallel algorithm. The row-parallel algorithm necessitates a pre-communication stage in which the input vector elements are communicated. Each $P_{k}$ sends the input vector elements that correspond to the nonzero column segments in off-diagonal blocks $A_{i k}, 1 \leq i \neq k \leq K$. This is also referred to as the expand operation since the same vector element can be sent to multiple processors. The vector elements that correspond to the columns which have at least one nonzero column segment in off-diagonal blocks (called coupling columns) necessitate expand operations. In Fig. B.10a, eight elements of the input vector ( $p[3], p[4], p[7], p[8], p[9], p[12], p[15], p[16])$ need to be communicated. For example, $P_{3}$ sends $p[12]$ to $P_{2}$ and $P_{4}$, which need this element in their local computations. On the other hand, the column-parallel algorithm necessitates a post-communication stage in which the partial results of the output vector elements are communicated. Each $P_{k}$ receives the output vector entries that correspond to the nonzero row segments in off-diagonal blocks $A_{k j}, 1 \leq j \neq k \leq K$. This is also referred to as the fold operation since the partial results for the same vector element can be received from multiple processors. The vector elements that correspond to the rows which have at least one nonzero row segment in off-diagonal blocks (called coupling rows) necessitate fold operations. In Fig. B.10b, eight elements of the output vector $(z[3], z[4], z[7], z[8], z[9], z[12], z[15], z[16])$ need to be communicated. For example, $P_{3}$ receives partial results for $z[12]$ from $P_{2}$ and $P_{4}$ to compute the final value of $z$ [12]. Observe that the communication of $p[12]$ in the row-parallel algorithm is the dual of the communication of $z[12]$ in the column-parallel algorithm.

It is possible to obtain 1D partitioning of sparse matrices using column-net and row-net hypergraph models. The details of these two models are given in Appendix C.

\section{Appendix C. Two computational hypergraph models for 1D partitioning}

There are several ways of obtaining a 1D row wise/column wise partitioning of coefficient matrix $A$. We briefly discuss two hypergraph models since they are central to the models proposed in this work. These models are also referred to as computational hypergraph models.

The column-net hypergraph model $\mathcal{H}_{\mathcal{R}}=\left(\mathcal{V}_{\mathcal{R}}, \mathcal{N}_{\mathcal{C}}\right)$ can be used to obtain a row wise partitioning of $A$ [31]. In this model, vertex set $\mathcal{V}_{\mathcal{R}}$ represents the rows of $A$ and net set $\mathcal{N}_{\mathcal{C}}$ represents the columns of $A$. There is a vertex $v_{i} \in \mathcal{V}_{\mathcal{R}}$ for each row $r_{i}$ and there is a net $n_{j} \in \mathcal{N}_{\mathcal{C}}$ for each column $c_{j}$. Net $n_{j}$ connects a subset of vertices that correspond to the rows that have a nonzero element in column $c_{j}$, i.e., $v_{i} \in n_{j}$ if and only if $a_{i j} \neq 0$. The weight $w_{i}$ of vertex $v_{i}$ is equal to the number of nonzeros in row $r_{i}$ and represents the computational load associated with $v_{i}$. 
The row-net hypergraph model $\mathcal{H}_{\mathcal{C}}=\left(\mathcal{V}_{\mathcal{C}}, \mathcal{N}_{\mathcal{R}}\right)$ can be used to obtain a column wise partitioning of $A$ [31]. In this model, vertex set $\mathcal{V}_{\mathcal{C}}$ represents the columns of $A$ and net set $\mathcal{N}_{\mathcal{R}}$ represents the rows of $A$. There is a vertex $v_{j} \in \mathcal{V}_{\mathcal{C}}$ for each column $c_{j}$ and there is a net $n_{i} \in \mathcal{N}_{\mathcal{R}}$ for each row $r_{i}$. Net $n_{i}$ connects a subset of vertices that correspond to the columns that have a nonzero element in row $r_{i}$, i.e., $v_{j} \in n_{i}$ if and only if $a_{i j} \neq 0$. The weight $w_{j}$ of vertex $v_{j}$ is equal to the number of nonzeros in column $c_{j}$ and represents the computational load associated with $v_{j}$.

Partitioning hypergraphs $\mathcal{H}_{\mathcal{C}}$ and $\mathcal{H}_{\mathcal{R}}$ with the objective of minimizing cutsize corresponds to minimizing total communication volume incurred in parallel sparse-matrix vector multiplication while maintaining the partitioning constraint on part weights corresponds to maintaining a balance on computational loads of processors.

\section{Appendix D. Communication hypergraph model for 1D partitioning}

The communication hypergraph (CHG) model [53] is a means of distributing communication tasks among processors with the aim of minimizing latency. A communication task is defined as a subset of processors that are involved in communicating a data object with a certain size. The CHG model strives to reduce the total number messages usually at the expense of increasing communication volume. However, although it increases the volume, it tries to obtain a balance on it. Reducing latency is a key factor to achieve scalability in large-scale systems as we show with our experiments. In this section, we review the CHG model for reducing latency overhead of 1D partitioned parallel $w=A p$ and $z=A^{T} r$ multiplies.

\section{D.1. Communication matrix}

As the first step, we form communication matrices $M_{R}$ and $M_{C}$ to summarize the communication requirements of rowparallel $w=A p$ and column-parallel $z=A^{T} r$, respectively. For row-parallel $w=A p$, let $p_{C}$ denote the $p$-vector elements that necessitate communication (via expand tasks). Communication matrix $M_{R}$ is then a $K \times\left|p_{C}\right|$ matrix where the rows of $M_{R}$ correspond to processors and the columns of $M_{R}$ correspond to expand communication tasks. In $M_{R}, m_{k j} \neq 0$ if and only if the corresponding coupling column $c_{j}$ has a nonzero column segment in the $k$ th row stripe of $A$. For example in $A$ (Fig. B.10a), column 12 has a nonzero at the second row stripe, thus there exists a nonzero at the corresponding entry in $M_{R}$ in Fig. D.11 at the intersection of row $P_{2}$ and column 12. The nonzeros of column $c_{j} \in M_{R}$ signify the set of processors that participate in communicating $p_{C}[j]$. The nonzeros of row $r_{k} \in M_{R}$ signify all expand tasks that $P_{k}$ takes part in. In Fig. D.11, the third row in $M_{R}$ has nonzero elements corresponding to columns 4, 12 and 15, indicating that $P_{3}$ is involved in communicating $p$ [4], $p$ [12] and $p$ [15]. Hence, a nonzero $m_{k j} \in M_{R}$ actually implies that $P_{k}$ participates in the communication of $p_{c}[j]$.

For column-parallel $z=A^{T} r$, let $z_{C}$ denote the $z$-vector elements that necessitate communication (via fold tasks). Communication matrix $M_{C}$ is then a $\left|z_{C}\right| \times K$ matrix where the rows of $M_{C}$ correspond to fold communication tasks and the columns of $M_{C}$ correspond to processors. In $M_{C}, m_{i k} \neq 0$ if and only if the corresponding coupling row $r_{i}$ has a nonzero row segment in the kth column stripe of $A^{T}$. For example in $A^{T}$ (Fig. B.10b), row 12 has a nonzero at the second column stripe, thus there exists a nonzero at the corresponding entry in $M_{C}$ in Fig. D.11 at the intersection of row 12 and column $P_{2}$. The nonzeros of row $r_{i} \in M_{C}$ signify the set of processors that participate in communicating $z_{C}[i]$. The nonzeros of column $c_{k} \in$

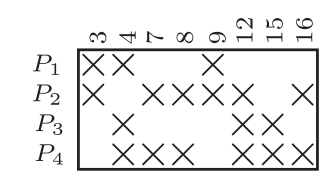

Communication matrix $M_{R}$

(for row-parallel $w=A p$ )
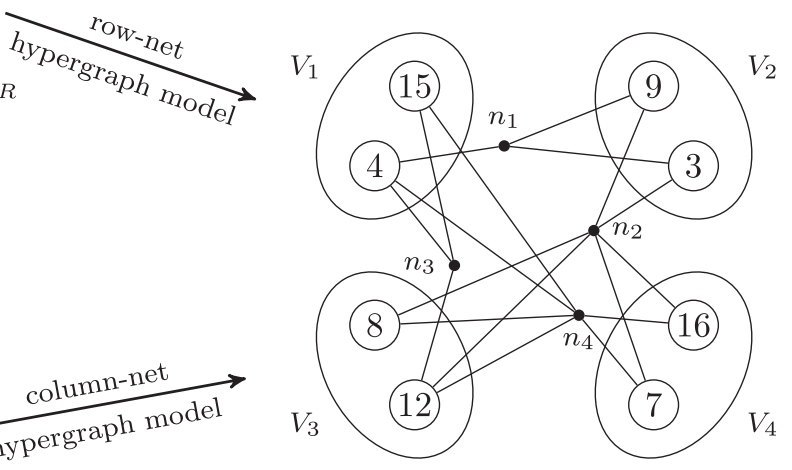

Communication hypergraph

vertices $=$ communication tasks nets $=$ processors

Communication matrix $M_{C}$

(for column-parallel $z=A^{T} r$ )

Fig. D11. Formation of the communication hypergraph from a communication matrix, and a four-way partition on this hypergraph. Matrices $M_{R}$ and $M_{C}$ summarize the communication requirements of $w=A p$ and $z=A^{T} r$ operations illustrated in Fig. B.10. 
$M_{C}$ signify all fold tasks that $P_{k}$ takes part in. In Fig. D.11, the third column in $M_{C}$ has nonzero elements corresponding to rows 4, 12 and 15, indicating that $P_{3}$ is involved in communicating $z[4], z[12]$ and $z[15]$. Hence, a nonzero $m_{i k} \in M_{C}$ actually implies that $P_{k}$ participates in the communication of $z_{C}[i]$.

\section{D.2. Formation of communication hypergraph}

The communication matrix is then used to form a hypergraph called communication hypergraph. We apply the row-net hypergraph model to communication matrix $M_{R}$ (vertices = columns, nets = rows) to obtain the communication hypergraph $\mathcal{H}_{R}^{C M}$ and we apply the column-net hypergraph model to communication matrix $M_{C}$ (vertices = rows, nets = columns) to obtain the communication hypergraph $\mathcal{H}_{C}^{C M}$. The vertex and net set of both hypergraphs are the same (see Fig. D.11). In both hypergraphs, nets correspond to processors (there are $K$ of them) and vertices correspond to communication tasks (there are $\left|p_{C}\right|=\left|z_{C}\right|$ of them). However, the semantics of these hypergraphs differ: the vertices in $\mathcal{H}_{R}^{C M}$ represent expand tasks in $w=A p$, while the vertices in $\mathcal{H}_{C}^{C M}$ represent fold tasks in $z=A^{T} r$. A net $n_{k}$ in both hypergraphs connects the set of vertices that correspond to communication tasks $P_{k}$ participates in. Each vertex $v_{i}$ is associated with a weight that signifies the volume of communication incurred by the corresponding expand or fold task. This value is generally equal to one less than the number of the nets $v_{i}$ is connected by, i.e., $d_{i}-1$.

\section{D.3. Partitioning of the communication hypergraph}

Obtaining a $K$-way partition $\Pi=\left\{\mathcal{V}_{1}, \mathcal{V}_{2}, \ldots, \mathcal{V}_{K}\right\}$ on $\mathcal{H}_{R}^{C M}$ or $\mathcal{H}_{C}^{C M}$ induces a communication task distribution for parallel matrix-vector or matrix-transpose-vector multiplies. Without loss of generality, assume that processor $P_{k}$ is associated with part $\mathcal{V}_{k}$. Expand or fold communication tasks represented by the vertices in $\mathcal{V}_{k}$ are assigned to $P_{k}$ by making this processor responsible for storing vector elements that necessitate these tasks. For instance in Fig. D.11, since $v_{12} \in \mathcal{V}_{3}, P_{3}$ is held responsible for storing $p[12]$ and $z[12]$, and expand and fold tasks necessitated by these elements. Consider a net $n_{k}$ in $\mathcal{H}_{R}^{C M}$ with the connectivity set $\Lambda\left(n_{k}\right)$. All parts except $\mathcal{V}_{k}$ in this set correspond to the processors that send a message to $P_{k}$, hence, $\lambda\left(n_{k}\right)-1$ (or $\lambda\left(n_{k}\right)$ if $\mathcal{V}_{k} \notin \Lambda\left(n_{k}\right)$ ) is equal to the number of messages $P_{k}$ receives. In a dual manner, consider the same net in $\mathcal{H}_{C}^{C M}$ again with the connectivity set $\Lambda\left(n_{k}\right)$. All parts except $\mathcal{V}_{k}$ in this set correspond to the processors that receive a message from $P_{k}$, hence, $\lambda\left(n_{k}\right)-1$ (or $\lambda\left(n_{k}\right)$ if $\mathcal{V}_{k} \notin \Lambda\left(n_{k}\right)$ ) is equal to the number of messages $P_{k}$ sends. In Fig. D.11, the connectivity sets of nets are as follows: $\Lambda\left(n_{1}\right)=\left\{\mathcal{V}_{1}, \mathcal{V}_{2}\right\}, \Lambda\left(n_{2}\right)=\left\{\mathcal{V}_{2}, \mathcal{V}_{3} \mathcal{V}_{4}\right\}, \Lambda\left(n_{3}\right)=\left\{\mathcal{V}_{1}, \mathcal{V}_{3}\right\}$ and $\Lambda\left(n_{4}\right)=\left\{\mathcal{V}_{1}, \mathcal{V}_{3}, \mathcal{V}_{4}\right\}$, making a total of $\left(\lambda\left(n_{1}\right)-1=1\right)+\left(\lambda\left(n_{2}\right)-1=2\right)+\left(\lambda\left(n_{3}\right)-1=1\right)+\left(\lambda\left(n_{4}\right)-1=2\right)=6$ messages. In [53], it is proven that partitioning a communication hypergraph with the aim of minimizing cutsize minimizes the total message count, while maintaining a balance among part weights preserves a balance on the communication volume.

By applying the CHG model, we obtain a different partition on rows and columns of the coefficient matrix and thus on its input and output space (for details, see Appendix F). Adopting a different partition finds its application in nonsymmetric sparse iterative solvers that allow distinct partitions on vectors and can be used to improve their scalability.

\section{Appendix E. Partitioning vectors in CGNE and CGNR solvers}

We describe why it is possible to use different partitions on the vectors used in CGNE and CGNR solvers. For other solvers, refer to [71]. We make the distinction between input and output space for the vectors in the solver. A vector is said to be in the input space of $A$ if it is multiplied with $A$ or it participates with the vectors in the input space of $A$ through linear vector operations. On the other hand, a vector is said to be in the output space of $A$ if it is obtained by multiplying $A$ with another vector or it participates with the vectors in the output space of $A$ through linear vector operations.

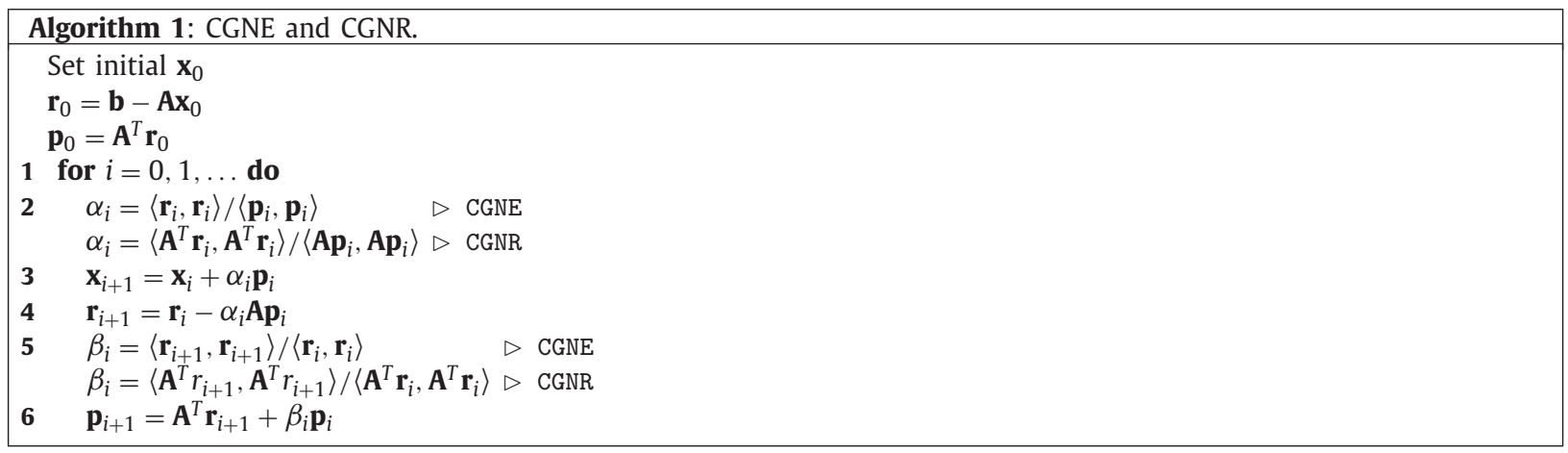

We present CGNE and CGNR algorithms in Algorithm 1. In each iteration of the solvers, there are two inner products, three SAXPY operations (for forming vectors $p, r, x$ ), one matrix-vector multiply of the form $w=A p$ and one matrix-transpose-vector multiply of the form $z=A^{T} r$. In $w=A p$, vectors $p$ and $w$ are in the input and output space of $A$, 

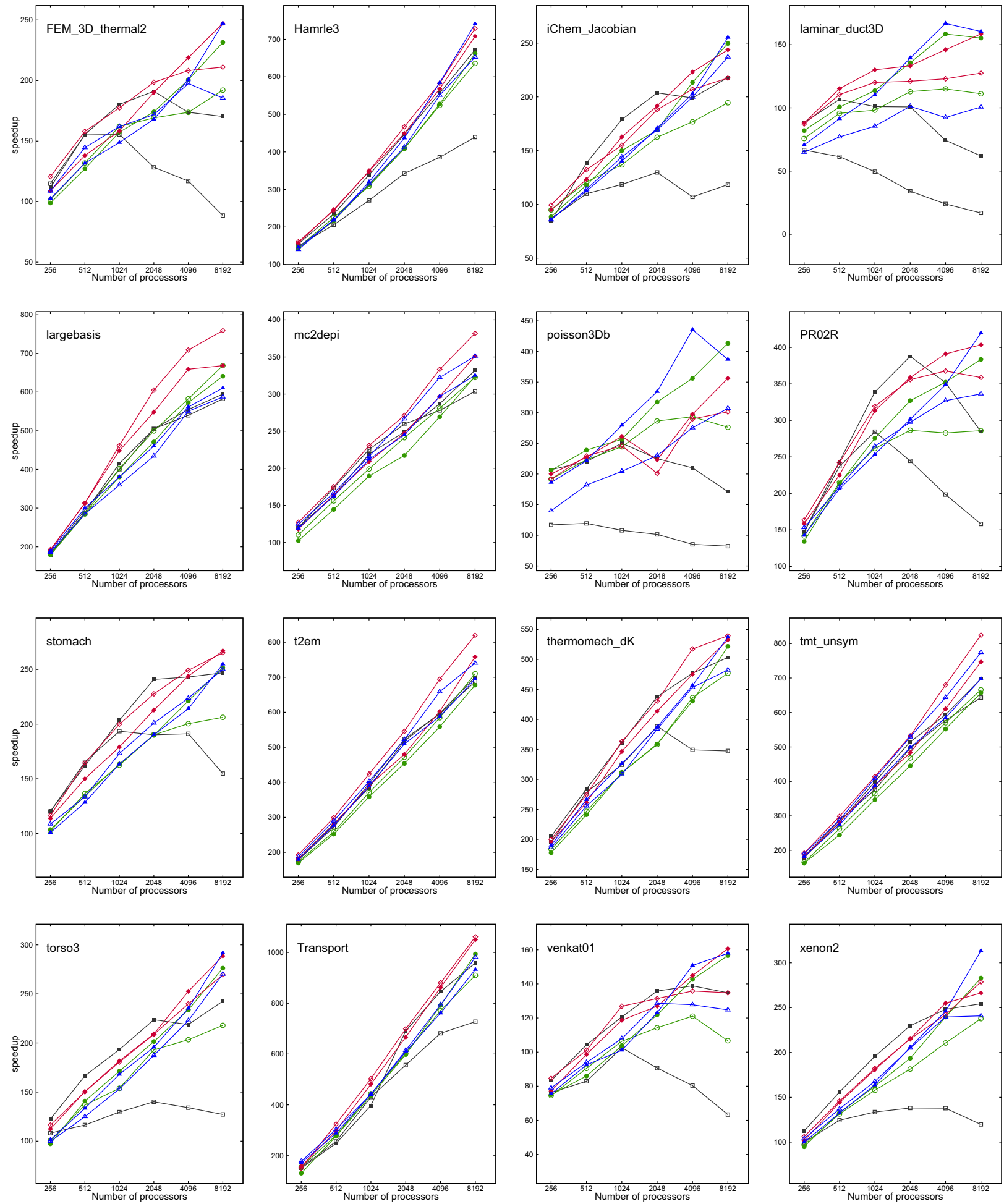

Fig. G12. Speedup curves for the remaining 16 matrices. 
respectively. In $z=A^{T} r$, vectors $r$ and $z$ are in the input and output space of $A^{T}$, respectively. Consider a row wise (column wise) partition of $A$. This induces a column wise (row wise) partition on $A^{T}$. Hence, the input space of $A$ coincides with the output space of $A^{T}$, and vice versa. This implies that the partition on vector $p$ is conformal with the partition on vector $z$, and the partition on vector $w$ is conformal with the partition on vector $r$. Since $x$ is involved in linear vector operations with vector $p$ (line 3 ), it should be partitioned conformally with vectors $p$ and $z$ to avoid unnecessary communication. As a result, we can have two distinct vector partitions in CGNE and CGNR: one on vectors $p, z$ and $x$, and another one on vectors $w$ and $r$.

\section{Appendix F. Obtaining different vector partitions using communication hypergraph model}

The communication hypergraph ( $\mathrm{CHG}$ ) model can be regarded as a post-processing phase to distribute the communication tasks. Although any partitioning model could be used, assume that the column-net computational hypergraph model is used in the first phase for $w=A p$ (Appendix C). As computational tasks are represented by vertices that correspond to rows of the matrix, partitioning this hypergraph actually induces a partition of the rows of the matrix. The result of this partitioning determines the communication tasks in the second phase. Applying the CHG in the second phase induces a partition of the columns of the matrix since communication tasks represented by the vertices are expand-type tasks performed on input vector elements. Hence, in the first phase, we minimize the communication volume by obtaining a partition of the rows, and in the second phase we minimize the number of messages by obtaining a different partition of the columns. An example of these two phases can be traced from Figs. B.10a and D.11. Assume that the result of the first phase is the partition obtained on $A$ in Fig. B.10a. Here, $P_{3}$ owns rows 8, 9, 10, 11, 12 and columns 10, 11, 12. The column partition is subject to change after applying the CHG model, which assigns vertices 8 and 12 to $\mathcal{V}_{3}$, thus assigning columns 8 and 12 to $P_{3}$. Since columns 10 and 11 do not necessitate communication, they are not included in the CHG model and directly assigned to $P_{3}$ at the end of the first phase. As a result, $P_{3}$ owns columns $8,10,11,12$. Hence, we obtained a nonsymmetric partition of the rows and columns assigned to $P_{3}$.

\section{Appendix G. Additional speedup curves}

We provided 12 speedup curves in Section 4.2. In this section, we provide 16 speedup curves that belong to the remaining matrices in Fig. G.12. Note that these curves do not change the findings of the paper and they are added here for the sake of completeness.

\section{References}

[1] D.A. Patterson, Latency lags bandwith, Commun. ACM 47 (2004) 71-75.

[2] S. L. Graham, M. Snir, C. A. Patterson (eds.), Getting Up to Speed, The Future of Supercomputing, The National Academies Press, 2006.

[3] J. Dongarra, M.A. Heroux, Toward a new metric for ranking high performance computing systems, Technical Report, SAND2013-4744, Sandia National Laboratories, 2013.

[4] H.M. Bücker, M. Sauren, A parallel version of the unsymmetric Lanczos algorithm and its application to QMR, Technical Report KFA-ZAM-IB-9606, Central Institute for Applied Mathematics, Research Center Julich, Germany, 1996.

[5] T.-X. Gu, X.-Y. Zuo, X.-P. Liu, P.-L. Li, An improved parallel hybrid bi-conjugate gradient method suitable for distributed parallel computing, J. Comput. Appl. Math. 226 (2009) 55-65.

[6] L. Yang, R.P. Brent, The improved BiCGStab method for large and sparse unsymmetric linear systems on parallel distributed memory architectures, in: Algorithms and Architectures for Parallel Processing, 2002. Proceedings. Fifth International Conference on, 2002, pp. 324-328, doi:10.1109/ICAPP.2002. 1173595.

[7] T.P. Collignon, M.B. van Gijzen, Minimizing synchronization in IDR(s), Numer. Linear Algebra with Appl. 18 (2011) $805-825$.

[8] A. Chronopoulos, C. Gear, S-step iterative methods for symmetric linear systems, J. Comput. Appl. Math. 25 (1989) $153-168$

[9] G. Meurant, Multitasking the conjugate gradient method on the CRAY X-MP/48, Parallel Comput. 5 (1987) $267-280$.

[10] E.F. D'Azevedo, V.L. Eijkhout, C.H. Romine, Conjugate gradient algorithms with reduced synchronization overheads on distributed memory processors, Technical Report, 56, Lapack Working Note, 1993.

[11] Y. Saad, Practical use of polynomial preconditionings for the conjugate gradient method, SIAM J. Sci. Statis. Comput. 6 (1985) 865-881.

[12] L.T. Yang, R.P. Brent, in: The Improved BiCG Method for Large and Sparse Linear Systems on Parallel Distributed Memory Architectures Proceedings of the 16th International Parallel and Distributed Processing Symposium, IPDPS '02, IEEE Computer Society, Washington, DC, USA, 2002, p. 315. http: //dl.acm.org/citation.cfm?id=645610.661567

[13] Z. Bai, D. Hu, L. Reichel, A newton basis GMRES implementation, IMA J. Numer. Anal. 14 (1994) $563-581$.

[14] A.T. Chronopoulos, S-step iterative methods for (non)symmetric (in)definite linear systems, SIAM J. Numer. Anal. 28 (1991) 1776-1789.

[15] A. Chronopoulos, C. Swanson, Parallel iterative S-step methods for unsymmetric linear systems, Parallel Comput. 22 (1996) 623-641.

[16] M. Hoemmen, Communication-avoiding Krylov Subspace Methods, Ph.D. thesis, Berkeley, CA, USA, 2010.AAI3413388.

[17] W.D. Joubert, G.F. Carey, Parallelizable restarted iterative methods for nonsymmetric linear systems. part I: Theory, Int. J. Comput. Math. 44 (1992) 243-267.

[18] E. Carson, N. Knight, J. Demmel, Avoiding communication in two-sided krylov subspace methods, Technical Report, UCB/EECS-2011-93, EECS Department, University of California, Berkeley,2011, http://www.eecs.berkeley.edu/Pubs/TechRpts/2011/EECS-2011-93.html.

[19] L. Grigori, S. Moufawad, Communication avoiding ILU0 preconditioner, Rapport de recherche, RR-8266, INRIA, 2013, http://hal.inria.fr/hal-00803250.

[20] J.W. Demmel, M.T. Heath, H.A. van der Vorst, Parallel numerical linear algebra, Acta Numer. 2 (1993) 111-197.

[21] E. de Sturler, H.A. van der Vorst, Reducing the effect of global communication in GMRES(m) and CG on parallel distributed memory computers, Appl. Numer. Math. 18 (1995) 441-459.

[22] T. Hoefler, P. Gottschling, A. Lumsdaine, W. Rehm, Optimizing a conjugate gradient solver with non-blocking collective operations, Parallel Comput. 33 (2007) 624-633.

[23] P. Ghysels, T. Ashby, K. Meerbergen, W. Vanroose, Hiding global communication latency in the GMRES algorithm on massively parallel machines, SIAM J. Sci. Comput. 35 (2013) C48-C71. 
[24] P. Ghysels, W. Vanroose, Hiding global synchronization latency in the preconditioned conjugate gradient algorithm, Parallel Comput. 40 (2014) 224-238.7th Workshop on Parallel Matrix Algorithms and Applications.

[25] L.C. Mcinnes, B. Smith, H. Zhang, R.T. Mills, Hierarchical Krylov and nested Krylov methods for extreme-scale computing, Parallel Comput. 40 (2014) $17-31$.

[26] O. Selvitopi, M. Ozdal, C. Aykanat, A novel method for scaling iterative solvers: Avoiding latency overhead of parallel sparse-matrix vector multiplies, 2014, doi: 10.1109/TPDS.2014.2311804.

[27] G. Ballard, J. Demmel, O. Holtz, O. Schwartz, Minimizing communication in numerical linear algebra, SIAM J. Matrix Anal. Appl. 32 (2011) 866-901.

[28] J. Demmel, M. Hoemmen, M. Mohiyuddin, K. Yelick, Avoiding communication in sparse matrix computations, in: Parallel and Distributed Processing, 2008. IPDPS 2008. IEEE International Symposium on, 2008, pp. 1-12, doi:10.1109/IPDPS.2008.4536305.

[29] M. Mohiyuddin, M. Hoemmen, J. Demmel, K. Yelick, Minimizing communication in sparse matrix solvers, in: Proceedings of the Conference on High Performance Computing Networking, Storage and Analysis, SC '09, pp. 36:1-36:12, ACM, New York, NY, USA, 2009, doi:10.1145/1654059.1654096.

[30] G. Karypis, V. Kumar, A fast and high quality multilevel scheme for partitioning irregular graphs, SIAM J. Sci. Comput. 20 (1998) $359-392$.

[31] U. Çatalyurek, C. Aykanat, Hypergraph-partitioning-based decomposition for parallel sparse-matrix vector multiplication, IEEE Trans. Parallel Distrib. Syst. 10 (1999) 673-693.

[32] F. Pellegrini, J. Roman, Scotch: a software package for static mapping by dual recursive bipartitioning of process and architecture graphs, in: H. Liddell, A. Colbrook, B. Hertzberger, P. Sloot (Eds.), High-Performance Computing and Networking, volume 1067 of Lecture Notes in Computer Science, Springer Berlin Heidelberg, 1996, pp. 493-498, doi:10.1007/3-540-61142-8_588.

[33] B. Vastenhouw, R.H. Bisseling, A two-dimensional data distribution method for parallel sparse matrix-vector multiplication, SIAM Rev. 47 (2005) 67-95.

[34] B. Hendrickson, T.G. Kolda, Graph partitioning models for parallel computing, Parallel Comput. 26 (2000) 1519-1534.

[35] W.J. Camp, S.J. Plimpton, B.A. Hendrickson, R.W. Leland, Massively parallel methods for engineering and science problems, Commun. ACM 37 (1994) 30-41.

[36] O.C. Martin, S.W. Otto, Partitioning of unstructured meshes for load balancing, Concurrency: Practice and Experience 7 (1995) 303-314.

[37] U. Çatalyurek, C. Aykanat, Decomposing irregularly sparse matrices for parallel matrix-vector multiplication, in: Proceedings of the Third International Workshop on Parallel Algorithms for Irregularly Structured Problems, IRREGULAR '96, Springer-Verlag, London, UK, 1996, pp. 75-86. http://dl.acm.org/ citation.cfm?id=646010.676990.

[38] C.-W. Ou, S. Ranka, Parallel incremental graph partitioning, Parallel and Distributed Systems, IEEE Transactions on 8 (1997) 884-896.

[39] A. Grama, G. Karypis, V. Kumar, A. Gupta, Introduction to Parallel Computing, 2nd, Addison-Wesley Longman Publishing Co., Inc., Boston, MA, USA, 2002.

[40] A. Ogielski, W. Aiello, Sparse matrix computations on parallel processor arrays, SIAM J. Sci. Comput. 14 (1993) 519-530.

[41] J.G. Lewis, R.A. van de Geijn, Distributed memory matrix-vector multiplication and conjugate gradient algorithms, in: Proceedings of the 1993 ACM/IEEE conference on Supercomputing, Supercomputing '93, ACM, New York, NY, USA, 1993, pp. 484-492, doi:10.1145/169627.169788.

[42] B. Hendrickson, R. Leland, S. Plimpton, An efficient parallel algorithm for matrix-vector multiplication, Int. J. of High Speed Comput. 7 (1995) $73-88$.

[43] A. Buluç, K. Madduri, Parallel breadth-first search on distributed memory systems, in: Proceedings of 2011 International Conference for High Performance Computing, Networking, Storage and Analysis, SC '11, ACM, New York, NY, USA, 2011, doi:10.1145/2063384.2063471.

[44] A. Yoo, A.H. Baker, R. Pearce, V.E. Henson, A Scalable Eigensolver for Large Scale-Free Graphs Using 2D graph partitioning Proceedings of 2011 International Conference for High Performance Computing, Networking, Storage and Analysis, SC'11, ACM, New York, NY, USA, 2011.pp. 63:1-63:11. http://doi.acm.org/10.1145/2063384.2063469

[45] U.V. Çatalyürek, Hypergraph Models for Sparse Matrix Partitioning and Reordering, 1999 Ph.D. thesis

[46] U. Çatalyürek, C. Aykanat, A fine-grain hypergraph model for 2D decomposition of sparse matrices, in: Proceedings of the 15th International Parallel \& Distributed Processing Symposium, IPDPS '01, IEEE Computer Society, Washington, DC, USA, 2001a, p. 118. http://dl.acm.org/citation.cfm?id=645609. 663255

[47] U. Çatalyürek, C. Aykanat, A hypergraph-partitioning approach for coarse-grain decomposition, in: Proceedings of the 2001 ACM/IEEE Conference on Supercomputing, SC '01, ACM, New York, NY, USA, 2001b, p. 28, doi:10.1145/582034.582062.

[48] B. Uçar, C. Aykanat, Minimizing communication cost in fine-grain partitioning of sparse matrices, in: A. Yazıcı, C. Sener (Eds.), Computer and Information Sciences - ISCIS 2003, volume 2869 of Lecture Notes in Computer Science, Springer Berlin Heidelberg, 2003, pp. 926-933, doi:10.1007/ 978-3-540-39737-3_115.

[49] U.V. Çatalyürek, C. Aykanat, B. Uçar, On two-dimensional sparse matrix partitioning: Models, methods, and a recipe, SIAM J. Sci. Comput. 32 (2010) 656-683.

[50] E.G. Boman, K.D. Devine, S. Rajamanickam, Scalable matrix computations on large scale-free graphs using 2D graph partitioning, in: Proceedings of the International Conference on High Performance Computing, Networking, Storage and Analysis, SC '13, ACM, New York, NY, USA, 2013, doi:10.1145/ 2503210.2503293.

[51] R.H. Bisseling, W. Meesen, Communication balancing in parallel sparse matrix-vector multiply, Electron. Trans. Numer. Anal. 21 (2005) 47-65.

[52] U.V. Çatalyürek, M. Deveci, K. Kaya, B. Uçar, UMPA: a Multi-objective, multi-level partitioner for communication minimization, in: D.A. Bader, H. Meyerhenke, P. Sanders, D. Wagner (Eds.), Graph Partitioning and Graph Clustering 2012, volume 588 of Contemporary Mathematics, AMS, 2013, pp. 53-66, doi:10.1090/conm/588/11704.

[53] B. Uçar, C. Aykanat, Encapsulating multiple communication-cost metrics in partitioning sparse rectangular matrices for parallel matrix-vector multiplies, SIAM J. Sci. Comput. 25 (2004) 1837-1859.

[54] R.W. Freund, G.H. Golub, N.M. Nachtigal, Iterative solution of linear systems, Acta Numerica 1 (1992) 57-100.

[55] N. Nachtigal, S. Reddy, L. Trefethen, How fast are nonsymmetric matrix iterations? SIAM J. Matrix Anal. Appl. 13 (1992) $778-795$.

[56] Y. Saad, Iterative Methods for Sparse Linear Systems, 2nd, Society for Industrial and Applied Mathematics, Philadelphia, PA, USA, 2003.

[57] H. Elman, Iterative methods for large, sparse, nonsymmetric systems of linear equations., Dissertation Abstracts Int. Part B: Sci. Eng. [DISS. ABST. INT. PT. B- SCI. \& ENG.], 43 (1982) 1982.

[58] R. Freund, N. Nachtigal, QMR: a quasi-minimal residual method for non-Hermitian linear systems, Numerische Mathematik 60 (1991) $315-339$.

[59] S. Balay, S. Abhyankar, M.F. Adams, J. Brown, P. Brune, K. Buschelman, V. Eijkhout, W.D. Gropp, D. Kaushik, M.G. Knepley, L.C. McInnes, K. Rupp, B.F. Smith, H. Zhang, PETSc Users Manual, Technical Report ANL-95/11 - Revision 3.5, Argonne National Laboratory, 2014. http://www.mcs.anl.gov/petsc.

[60] G. Karypis, R. Aggarwal, V. Kumar, S. Shekhar, Multilevel hypergraph partitioning: applications in VLSI domain, IEEE Trans. Very Large Scale Integr. Syst. 7 (1999) 69-79.

[61] C. Aykanat, B.B. Cambazoglu, B. Uçar, Multi-level direct k-way hypergraph partitioning with multiple constraints and fixed vertices, J. Parallel Distrib. Comput. 68 (2008) 609-625.

[62] E. Horowitz, S. Sahni, Fundamentals of Computer Algorithms, Computer Science Press, Rockville, MD, USA, 1978.

[63] T.A. Davis, Y. Hu, The University of Florida sparse matrix collection, ACM Trans. Math. Softw. 38 (2011) 1:1-1:25.

[64] G. Karypis, V. Kumar, Multilevel k-way hypergraph partitioning, in: Proceedings of the 36th Annual ACM/IEEE Design Automation Conference, DAC '99, ACM, New York, NY, USA, 1999, pp. 343-348, doi:10.1145/309847.309954.

[65] H.D. Simon, S.-H. Teng, How good is recursive bisection? SIAM J. Sci. Comput. 18 (1997) 1436-1445.

[66] E.D. Dolan, J.J. Moré, Benchmarking optimization software with performance profiles, Math. Program. 91 (2002) $201-213$.

[67] C. Berge, Graphs and Hypergraphs, Elsevier Science Ltd, 1985.

[68] C. Aykanat, A. Pinar, U.V. Çatalyürek, Permuting sparse rectangular matrices into block-diagonal form, SIAM J. Sci. Comput. 25 (2004) $1860-1879$.

[69] B. Uçar, C. Aykanat, Revisiting hypergraph models for sparse matrix partitioning, SIAM Rev. 49 (2007) $595-603$.

[70] T. Lengauer, Combinatorial Algorithms for Integrated Circuit Layout, John Wiley \& Sons, Inc., New York, NY, USA, 1990.

[71] B. Uçar, C. Aykanat, Partitioning sparse matrices for parallel preconditioned iterative methods, SIAM J. Sci. Comput. 29 (2007) 1683-1709. 